\title{
Gradhiva
}

GRADHIV

Revue d'anthropologie et d'histoire des arts

13 | 2011

Pièges à voir, pièges à penser

\section{Images mémorables pour un texte immuable}

Les catéchismes pictographiques testériens (Mexique, $\mathrm{XVI}^{\mathrm{e}}$-XIX ${ }^{\mathrm{e}}$ siècles)

Memorable images for an immutable text. Pictographic Testerian catechisms

(16th to 19th Mexico)

\section{Bérénice Gaillemin}

\section{(2) OpenEdition}

Journals

Édition électronique

URL : http://journals.openedition.org/gradhiva/2068

DOI : 10.4000/gradhiva.2068

ISSN : $1760-849 X$

Éditeur

Musée du quai Branly Jacques Chirac

Édition imprimée

Date de publication : 18 mai 2011

Pagination : 204-225

ISBN : 978-2-35744-042-5

ISSN : 0764-8928

Référence électronique

Bérénice Gaillemin, «Images mémorables pour un texte immuable », Gradhiva [En ligne], 13 | 2011, mis en ligne le 18 mai 2011, consulté le 21 septembre 2020. URL : http://journals.openedition.org/ gradhiva/2068; DOI : https://doi.org/10.4000/gradhiva.2068

(c) musée du quai Branly 


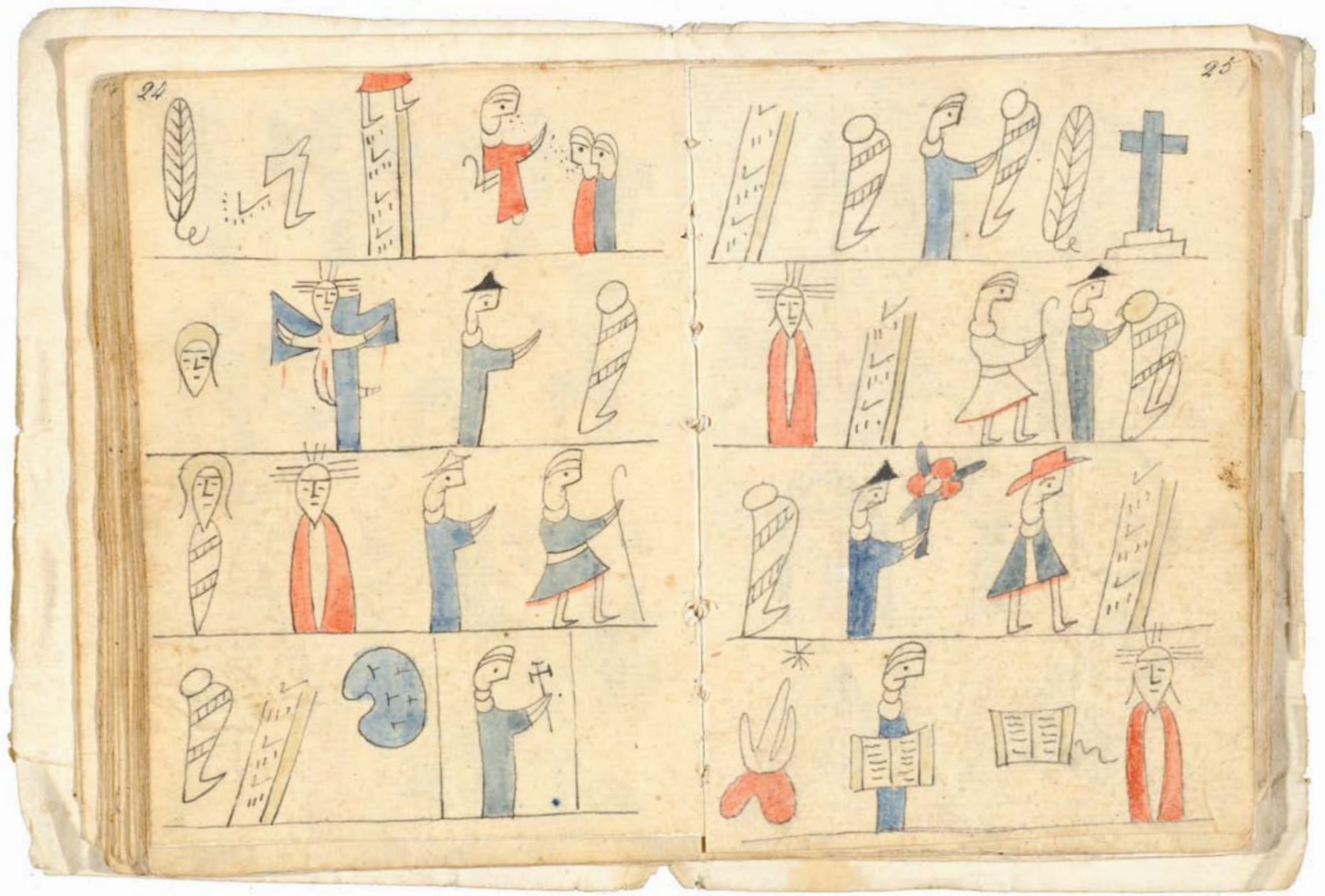

Testérien 833, Garrett Mesoamerican Ms. 3a, fol. 24V25r. Manuscripts Division, Department of Rare Books and Special Collections, Princeton University. 


\section{Images mémorables pour un texte immuable Les catéchismes pictographiques testériens (Mexique, $x V^{e}-x^{e} x^{e}$ siècles)}

Bérénice Gaillemin

\footnotetext{
-

1. À la différence de ce que propose l'école «galarzienne » initiée par Joaquín Galarza (Galarza et Monod 1980) et suivie, en ce qui concerne les testériens, par de nombreux auteurs (par exemple Balmaseda 1989, Bonilla 2004 et Bernand 2009), nous ne souhaitons pas proposer d'autres lectures des images que celles des textes fixes du catéchisme.
}

2. La langue nahuatl, ancienne langue dite des Aztèques, avait été choisie comme langue véhiculaire tant par les Mexicas au sein de leur empire que par les nouveaux conquistadors espagnols. Fonctionnant comme lingua franca, elle continuait de servir pour l'évangélisation et l'éradication de l'idolâtrie. Parmi les traductions du Notre Père en nahuatl, nous avons choisi ici d'utiliser la version contenue dans la Doctrina cristiana du franciscain Alonso de Molina (1941 [1546]).
Les catéchismes testériens sont de petits cahiers dans lesquels ont été transcrites au moyen d'images les prières principales et les différentes listes de préceptes que les catéchumènes devaient apprendre par cœur dans le cadre de ce que l'on nomme désormais la conquête spirituelle du Mexique. Ils offrent donc une occasion exceptionnelle d'étudier les rapports complexes entre image, langue et parole, car ces images ne se contentent pas d'illustrer des textes, mais les transcrivent de manière précise.

En effet, puisque les catéchismes pictographiques ont pour fonction de transmettre un texte fixe, et disponible sous forme écrite au moyen de caractères latins - celui du catéchisme -, il est possible d'évaluer le rapport qu'entretiennent les images avec les mots auxquels elles sont censées correspondre ${ }^{1}$. Afin de déterminer quels mots les images encodent et si ce processus s'enracine dans une langue en particulier, nous utilisons les versions espagnoles de la prière et les traductions en langue nahuatl établies par différents missionnaires dès le $\mathrm{xvI}^{\mathrm{e}}$ siècle ${ }^{2}$. Ces textes en caractères latins, imprimés et diffusés sur place grâce à l'importation de l'imprimerie autour de 1537 en Nouvelle-Espagne (Gil 1993 : 274), correspondent à la version idéale qui devait être mémorisée par les catéchumènes. La comparaison entre les catéchismes écrits et les catéchismes pictographiques permet de montrer que certaines images transcrivent non seulement des substantifs et des verbes, mais aussi - quoique plus exceptionnellement - des adverbes et autres locutions, ainsi que les marques du pluriel et de la négation. L'ancrage dans le nahuatl est mis en évidence par l'usage de procédés phonographiques (de type rébus), même si certaines images s'en émancipent : dans de tels cas, c'est la syntaxe - nahuatl ou castillane - qui permet de déterminer dans quelle langue était le texte original.

Notre approche tranche donc avec les analyses antérieures, qui privilégient l'étude de l'iconographie et du symbolisme, et qui, postulant que les auteurs de ces carnets sont peut-être d'anciens tlàcuiloquê, les scribes de l'ancien Mexi- 
que dont l'art d'écrire-peindre ${ }^{3}$ au moyen de pictographies a perduré jusqu'au XVIII ${ }^{e}$ siècle, voient dans les traditions indigènes l'origine de certaines images testériennes (Galarza et Monod 1980). Parmi les «traits indigènes », observables dans les écrits de tradition pictographique précolombienne, sont alors énumérés l'absence de profondeur, les couleurs uniformes des fonds, le goût prononcé pour la représentation de têtes de profil ou le principe pars pro toto (qui procède par synecdoque : une plume pour un oiseau par exemple), ou encore des glyphes caractéristiques des codices, tels que les sacs d'encens, les plumes, les momies, les volutes de la parole, la notation des nombres au moyen de cercles-unités et les fleurs.
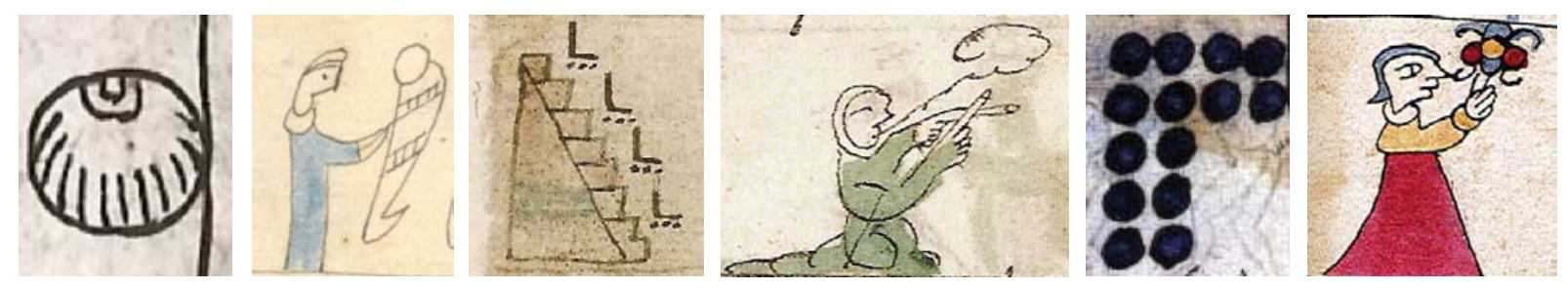

Plume, momie ou paquet funéraire, traces de pas, volute de la parole, nombre 14 et fleur dans les testériens $817,833,810,807,813$ et 814 . (Voir les références des illustrations en annexe, p. 224.)

Néanmoins, il est possible que ces images aient été élaborées par des missionnaires, et l'emploi de l'épithète " testérien » depuis la fin du xix siècle (Aubin 1885) contribue à diffuser cette idée. Le missionnaire franciscain Testera, imité ensuite par ses coreligionnaires, aurait créé ces outils afin de communiquer facilement avec les populations nouvellement conquises et de les instruire au plus vite. La question reste aujourd'hui ouverte mais il est difficile d'y répondre en ne s'intéressant qu'à l'iconographie. En effet, de nombreuses images issues du répertoire mexicain existaient à la même époque dans l'Ancien Monde : la volute de la parole aztèque peut tout à fait être associée aux phylactères qui sortaient de la bouche de Dieu dans les scènes de création du monde, et l'image de l'enfer, associée parfois à la déesse de la terre aztèque, Tlaltecuhtli, trouve son équivalent dans les représentations européennes du Léviathan. Ne sachant pas qui a peint ces images, il est difficile de leur octroyer une fonction symbolique déterminée.

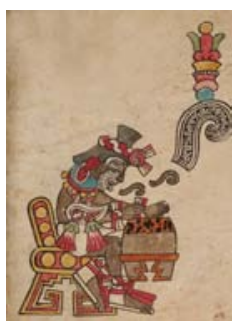

a

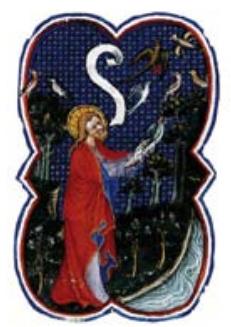

b

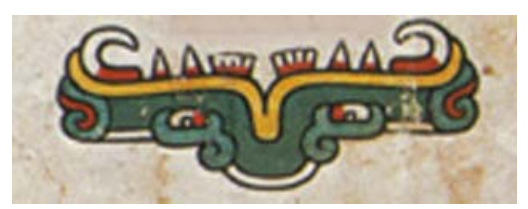

C

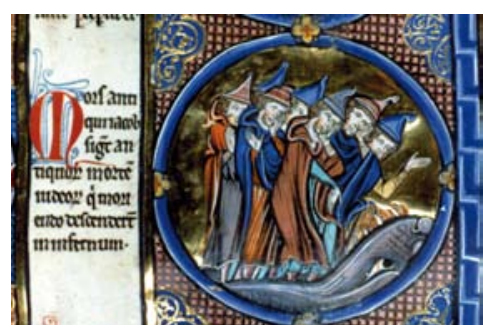

d

Volute de la parole et monstre de la terre dans les codices traditionnels (a. Codex Borbonicus et c. Codex Fejérváry-Mayer) et les manuscrits médiévaux (b. Bible historiale et d. Bible moralisée).

3. Le mot tlàcuilo (pl. tlàcuiloquê) provient du verbe nahuatl ìcuiloa qui, comme graphein en grec, peut être indifféremment traduit par «écrire » ou «peindre ».
En effet, les données contextuelles concernant ces outils missionnaires originaux restent extrêmement lacunaires. La datation des testériens demeure la question la plus problématique. Hétérogène, le corpus est constitué d'une 
trentaine de manuscrits conservés dans différentes institutions en Europe, au Mexique et auxÉtats-Unis. Tous ces catéchismes, que l'on désignera par le numéro attribué par John Glass (1975) et Anne Whited Normann (1985), possèdent des caractéristiques communes telles que leur petite taille (format de poche) et leur contenu catéchétique, transmis au moyen d'un enchaînement de petites images disposées les unes à la suite des autres par bandes horizontales. Ces carnets sont généralement considérés comme d'authentiques manuscrits élaborés au lendemain de la prise de Mexico-Tenochtitlan, dès l'arrivée des premiers ordres mendiants. Pourtant, seuls deux d'entre eux peuvent être datés du Xvi siècle, et plutôt de la fin du siècle (806 et 810). D’autres auraient été élaborés à la fin du $\mathrm{xVII}^{\mathrm{e}}$, tout au long du xvIII ${ }^{\mathrm{e}}$ ou bien encore au xix siècle. Prenant en compte le phénomène de la copie, Normann a effectué des regroupements ${ }^{4}$ : l'auteur postule l'existence d'un prototype, aujourd'hui perdu, dont dériveraient tous les membres de chaque groupe. Elle compte donc au total onze types différents dont chaque "prototype » daterait effectivement du xvi ${ }^{e}$ siècle ${ }^{5}$. Nous avons ailleurs souligné l'hétérogénéité du contenu catéchétique de chacun des carnets (Gaillemin 2010 et à paraître ${ }^{6}$ ), dont l'étude précise fournit des indices de datation en même temps qu'elle renseigne sur l'ordre religieux dans le giron duquel ont pu circuler de tels outils d'évangélisation.

S'intéresser à la technique scripturale plus qu'au style, à l'iconographie ou au symbolisme testérien, permet de contribuer aux débats sur l'origine et le contexte de production de ces catéchismes. Mais surtout, notre approche met en relief le caractère expérimental de la création de tels outils missionnaires, élaborée à partir d'essais, de réajustements et d'innovations ${ }^{7}$; nous pouvons dès lors décrire les différents procédés employés, souligner l'hétérogénéité des solutions (dans la lignée de Normann 1985 et Resines 2007), la variété des langues de lecture, et considérer les dynamiques de transformation sémiotique qui opèrent d'un cahier à l'autre. Il s'agit d'un surprenant laboratoire pour enrichir notre compréhension des rapports entre discours et images, essentiellement dans la perspective d'une relation sémiotique, mais aussi d'une relation mnémonique. En effet, en prenant l'exemple du procédé phonographique, nous envisagerons finalement le degré de sollicitation mémorielle auquel les lecteurs sont soumis lors de la phase de lecture, en montrant que les images testériennes peuvent jouer le rôle d'embrayeurs mémoriels.

\section{Les logiques logographiques : du lexique à la grammaire}

La majorité des images employées peuvent se lire dans toutes les langues. Ces "logogrammes" correspondent à un mot ou à un groupe de mots dont on trouve l'équivalent dans les textes imprimés, et il est possible de les distinguer en fonction de leur degré d'iconicité (images plus ou moins abstraites). Le mot peut être facilement représentable : c'est le cas par exemple du mot "pain ", écrit avec l'image d'une tortilla, galette de maïs de forme circulaire à la base de l'alimentation de nombreuses sociétés mésoaméricaines. Un mot plus abstrait peut être écrit au moyen d'une image symbolique : on rencontre alors parfois l'image d'une fleur pour écrire le mot "grâce" ou le verbe "estimer ". Ces images fonctionnent par extension du signifiant. Abstraites ou concrètes, elles ne se réfèrent pas directement à ce qu'elles représentent. Dans ce cas, l'image du soleil ne sert plus à écrire le mot «soleil», mais le mot «jour» ou «aujourd'hui », celle d'une

\footnotetext{
- $\odot$

4. Il existerait cinq grands groupes qu'elle nomme groupes Gante, Egerton, Sahagún, García Icazbaleceta et Tulane-Brown, ainsi que six exemplaires dits «isolés 》.

5. L'existence de ces «prototypes» ne pourra jamais être fermement prouvée. Concernant la datation, les analyses de filigranes effectuées par Batalla (à paraître) permettent d'affirmer que les manuscrits les plus représentatifs du corpus s'avèrent beaucoup moins anciens que l'on ne le suppose généralement. Par ailleurs, il est possible que certains exemplaires aient été confectionnés dans les ateliers des falsifications de codices œuvrant à la fin du xvI et au début du siècle suivant.

6. Par exemple, alors que le 817, composé d'une seule planche, donne seulement la liste des articles de la Foi et celle des commandements de la loi de Dieu, on trouve dans les quarante feuilles du 808 les deux mêmes listes, accompagnées des cinq prières principales (signe de croix, Pater noster, Ave Maria, Salve regina et Credo], le tout enrichi du Confiteor, d'un petit dialogue en questions-réponses puis de la liste des commandements de l'Église, des sacrements, des œuvres de miséricorde et des béatitudes.

7. Bien que les écarts de datation puissent avoir des répercussions considérables sur la technique mise en œuvre dans les carnets, nous ne connaissons pas la date des éventuels prototypes. En conséquence, nous ne ferons pas ici de distinction entre les différents manuscrits en termes de chronologie.
} 
"volute sortant de la bouche " permet quant à elle d'écrire le substantif « règne ». Dans la prière du Notre Père, différentes images correspondent aux substantifs " père ", « nom ", « règne ", " volonté », " péché », «tentation », « mal ", aux adverbes « aujourd'hui » et "quotidien», ainsi qu'aux verbes «être » (verbe d'état), " sanctifier », "venir », « faire », « donner », " pardonner », « offenser », « délivrer ».

Lorsqu'il s'agit d'illustrer la descente de Dieu le Père sur terre, le verbe "venir » (dans la phrase "que Ton règne vienne") est figuré par la représentation d'un personnage ou bien de deux jambes sur une échelle (806, 809 et 808). Parfois, on trouve seulement des traces de pas qui, dans les codices dits traditionnels, indiquent un mouvement, un chemin, parfois une ascendance généalogique (810 et 814). Le logogramme "règne" complète la phrase : ou bien Dieu est représenté assis en majesté, une fleur ou un sceptre cruciforme à la main, ou bien ce sont les symboles de son pouvoir (la couronne ou la parole) que le peintre a choisi de représenter.
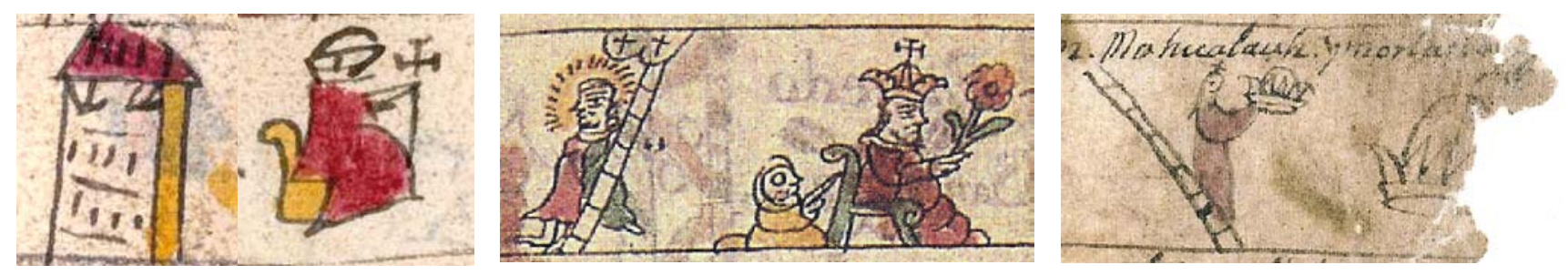

806, 809 et 808 : échelle («venir ») + Dieu en majesté/couronne (« règne »).
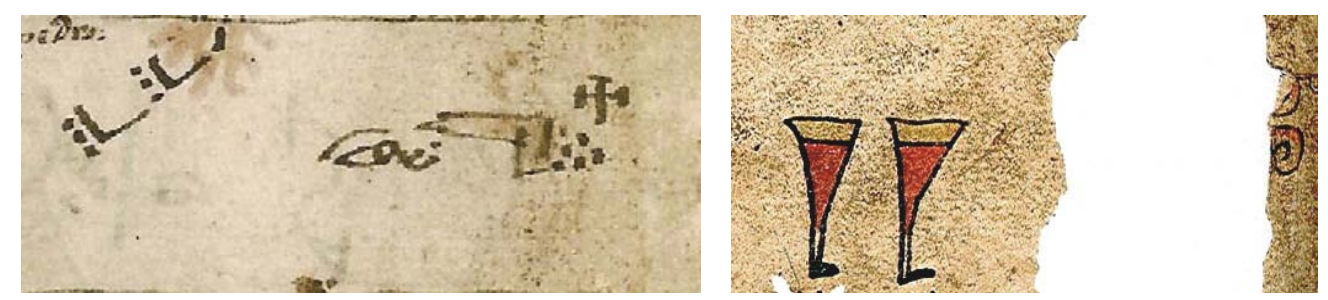

810 et 814 : traces de pas/jambes («venir ») + volute de la parole (« règne »). "Que vienne Ton règne."

- $\bigcirc$

8. Dans le 810 , la volute est de forme angulaire et se termine par de petits points surmontés d'une croix (le règne du Christ). Elle est précédée d'un œil que nous décrirons plus loin. Dans le 814 , bien que le manuscrit soit lacunaire, il est possible de distinguer les contours arrondis de la volute.
Pour figurer le «nom», qui renvoie principalement au nom de Dieu, les solutions varient aussi d'un peintre à l'autre. Dans les 806 et 813 , le mot correspond à un signe quadrangulaire, sorte de cartouche dans lesquelles sont inscrites, de façon parfois maladroite, les lettres INRI (Iesus Nazarenus Rex Iudæorum) correspondant à l'acronyme du Christ. Suivant le même procédé, ce mot a été écrit dans le 807 au moyen du monogramme IHS (pour Iesus Hominum Salvator). Seule la lettre I, ornementée à la façon de certaines lettrines médiévales, a été choisie pour désigner le substantif dans le 808. Dans le 810, il a été écrit au moyen d'un D majuscule inversé : cette première lettre de Dios pouvait être lue «nom ", par association d'idées (et s'apparente également aux symboles typographiques "pieds de mouche" dont la fonction était de mettre en relief le début d'un chapitre ou d'un paragraphe). Une autre image, plus abstraite, consiste en une croix circonscrite par un cercle (823, groupe Icazbalceta). Enfin, le scribe du 809 a représenté ce qui semble s'apparenter à un rouleau de papier. Dans les 806, 808 et 810 , 
le même logogramme est employé indistinctement pour écrire non seulement le «nom» (de Dieu), mais aussi le substantif "nom», par exemple lorsque les «noms » des différents sacrements sont énumérés.
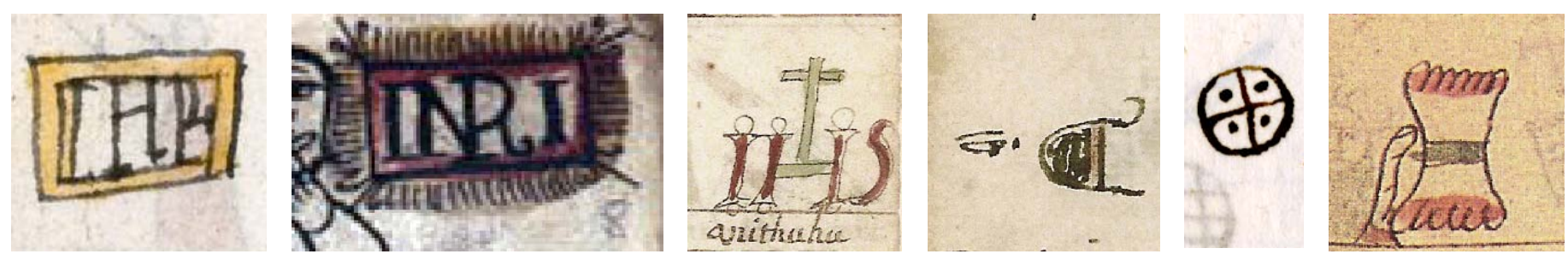

Le mot «nom » dans les exemplaires $806,813,807,808,810,823$ et 809 .

Mis à part ces logogrammes, d'autres images ont servi à écrire des mots plus difficilement représentables. En effet, outre les substantifs et verbes pour lesquels des solutions graphiques ont été trouvées, la prière du Notre Père contient d'autres lemmes ayant posé un problème lors de leur écriture en image. "Notre »,

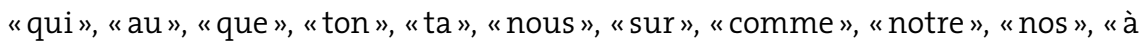
ceux", "ne nous... pas", " de tout ", "ainsi soit-il » font pourtant partie intégrante de la prière et devaient être mémorisés. Dans les testériens, "qui», «au », «ton, ta" et "sur" ne semblent pas avoir retenu l'attention des scribes, mais l'écriture d'autres mots s'est en revanche révélée indispensable. Par exemple, dans la phrase " et pardonnez-nous nos péchés comme nous pardonnons à ceux qui nous ont offensés", alors que le peintre du 806 n'a écrit que les mots "pardonner " (deux fois), "offenses» et "offenser», celui du 809 a en outre précisé les mots «et », « à nous », « nos », « comme », « nous » et « nos ».

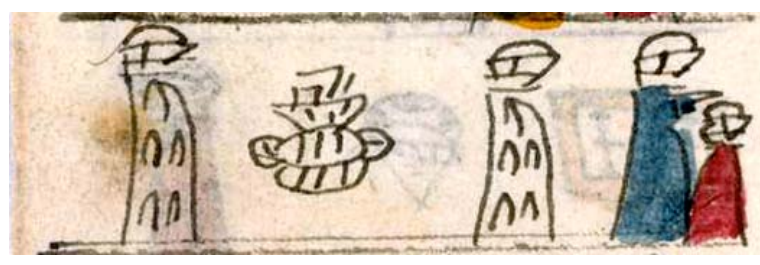

«[... et] pardonnez[-nous nos] offenses [comme nous] pardonnons [à ceux qui nous ont] offensés » (806).

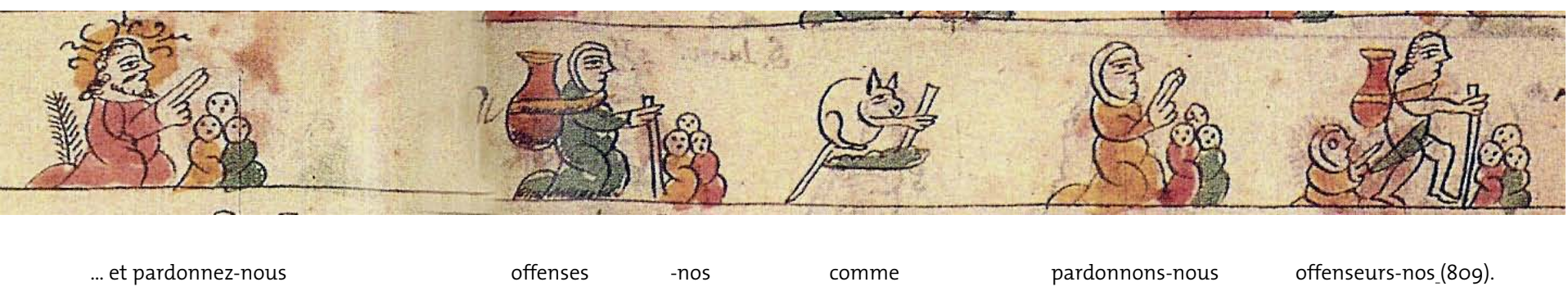


- $\circ$

9. On notera néanmoins que, pour l'écriture des toponymes, les suffixes locatifs «sous », «sur», «près de », etc., ont été encodés.

10. Beaucoup moins régulier, un procédé comparable est employé dans le 813, avec seulement deux personnages représentés.

11. Pour faciliter la lecture de cet article, nous avons pris le parti d'écrire les phonèmes au moyen de caractères latins. Ainsi, selon l'écriture carochienne normalisée (établie à partir de la grammaire du jésuite Horacio Carochi, Arte de la lengua mexicana publiée en 1645 [2001] ], la « consonne » ch (contenue dans tech) correspond au phonème $/ \mathrm{tch} /$, $x$ au son $/ \mathrm{ch} /$, uh ou hu au son $/ w /$, cu ou uc au son /kw/, z au son /s/, tz au son /ts/ et $c$, suivant la voyelle devant laquelle elle est placée, aux sons $/ \mathrm{k} /$ et $/ \mathrm{s} /$.
Le degré de précision varie donc, certes, en fonction des peintres, mais cette façon d'enregistrer certaines subtilités du langage (l'adverbe «comme» par exemple) est propre à l'écriture testérienne. En effet, ces lemmes - adverbes, conjonctions, pronoms personnels ou possessifs - n'ont jamais été encodés dans les manuscrits mexicains précolombiens ou coloniaux, dont les logogrammes se limitent aux noms propres (anthroponymes et toponymes ${ }^{9}$ ), dates et nombres. L'écriture d'adverbes ou de mots de liaison encodant la syntaxe des propositions - et pas seulement des syntagmes nominaux ou verbaux-s'impose donc comme une innovation des testériens. Ce caractère expérimental explique sans doute que les scribes n'aient pas porté leur choix sur les mêmes mots, et que leurs solutions soient disparates.

Si le temps des verbes conjugués n'est généralement pas encodé, certains signes sont néanmoins employés afin de préciser la formation grammaticale d'un mot. Lorsque les verbes, les pronoms possessifs ou les compléments d'objet se trouvent à la première personne du pluriel, le peintre du 809 a notamment choisi de figurer un groupe de trois personnages représentés de face ${ }^{10}$ : c'est le cas des logogrammes complexes "pardonnez-nous", "nos offenses", "nous pardonnons" dans lesquels le complément d'objet «nous», le possessif «notre» et le sujet "nous » sont indiqués, invariablement écrits avec le même petit groupe. Soustelle l'a d'ailleurs remarqué au sujet du manuscrit 807, mais, parce que ce catéchisme contient des gloses en langue otomi, il a traduit l'image du petit groupe par la racine du pluriel [-ho], affirmant que «ce symbole est à la fois figuratif et phonétique» (1936:16). Toutefois les phonèmes, s'ils semblent être identiques en otomi, sont différents en nahuatl (/tetch/, /to/ et /ti/ $\left.{ }^{11}\right)$ et en espagnol (nuestra, nosotros ou nos) selon qu'il s'agit d'un complément d'objet, d'un possessif ou d'un sujet. L'image n'est donc pas phonétique; elle oriente la lecture, permet de mieux conjuguer et s'apparente au signe formé par trois traits verticaux utilisé dans l'écriture de l'ancienne Égypte pour marquer aussi le pluriel. La fonction de ce signe est celle de « déterminatif-pluriel».
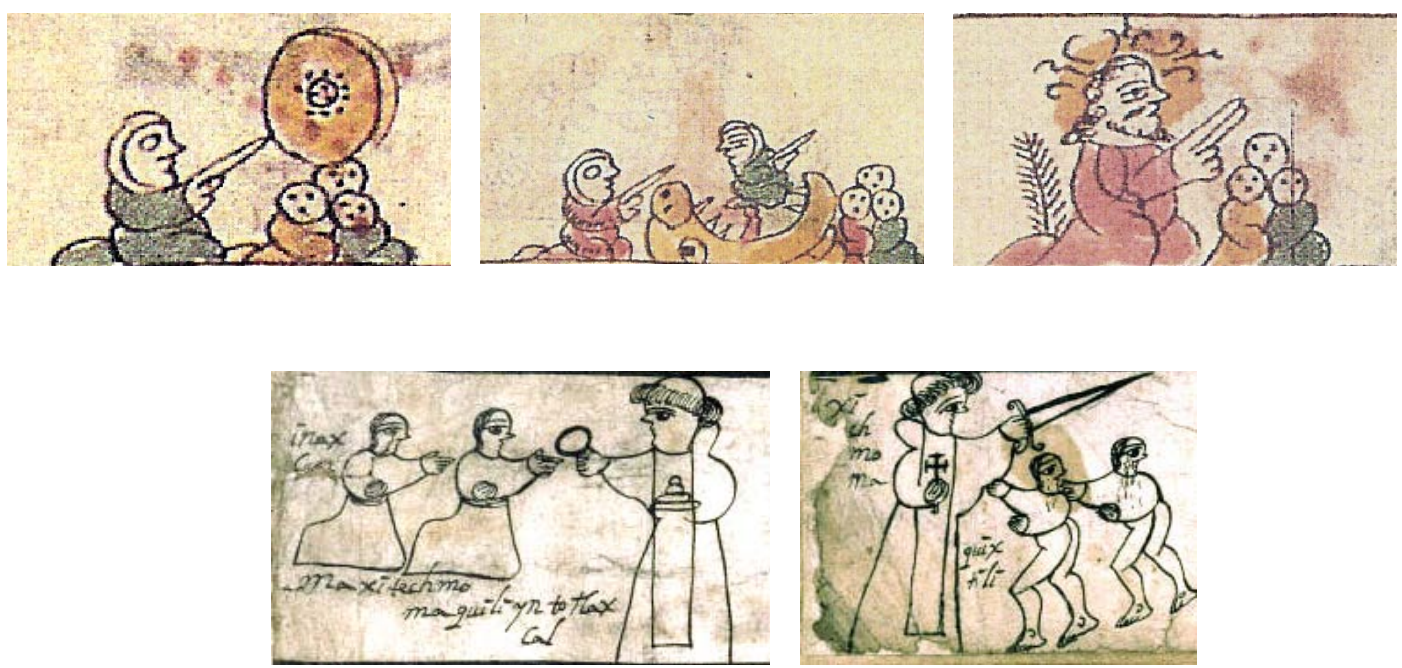

La notation du pluriel : " notre tortilla », « nous ne tomberons pas ", «pardonne-nous » (809) et « donne-nous », « délivre-nous» (813). 
Un autre procédé est le "déterminatif-négation ", qui consiste à encoder une phrase à la forme négative en orientant la tête des personnages dans le sens inverse de celui de la lecture ${ }^{12}$. Pour le Notre Père, il n'a été utilisé que dans trois testériens $\left(809,808\right.$ et $\left.813^{13}\right)$ et notamment pour écrire le mot "mal ", traduit en nahuatl par une forme négative. Le «mal», c'est-à-dire ce qui n'est pas bon, a donc été mis en image par le peintre du 809 au moyen d'un personnage tournant la tête (ce geste signifiant "ne... pas", àmo), tenant dans ses mains un vase rempli de fleurs symbolisant le «bon» (cualli). On observe le même procédé dans les propositions du décalogue énoncées à la forme négative.

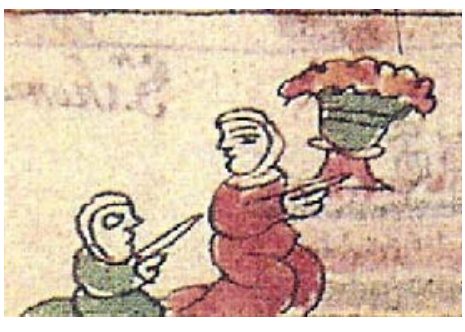

a

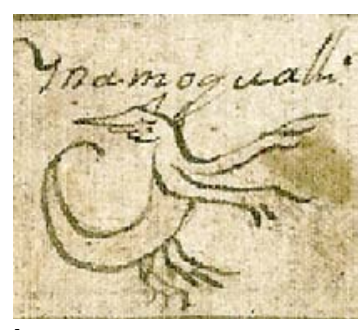

b

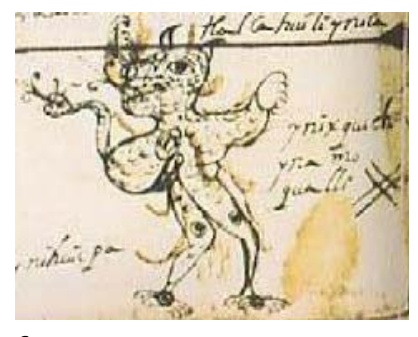

C

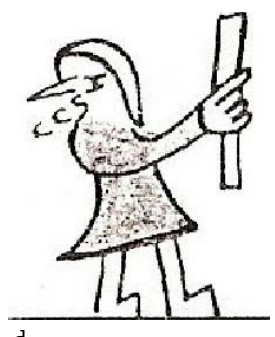

d

La marque de la négation dans les 809, 808, 813 (mot «mal ») et 830 (" tu ne tueras point »).

À notre connaissance, l'usage d'un tel procédé est propre aux testériens, ce qui peut s'expliquer si l'on considère que, dans les codices traditionnels, le sens de lecture n'était pas unique et les images ne correspondaient pas à un texte fixe et invariable : elles étaient en effet disposées dans différentes directions «afin de créer des messages visuels qui redoublaient parfois la parole mais ne l'enregistraient généralement pas » (Boone 2007 : 33). En revanche, dans les catéchismes, les images sont alignées dans des bandes horizontales et se lisent généralement les unes après les autres, ce qui permet de jouer plus facilement sur leur orientation.

Cependant, dans les manuscrits 808 et 813, c'est directement la figure allégorique du mal, un monstre griffu à la queue recourbée, qui tourne la tête dans le sens inverse de celui de la lecture. Cette contorsion rappelle celle de certains personnages ou divinités des manuscrits précolombiens : ainsi, la pose de ceux « dont les jambes sont repliées, le corps légèrement penché en avant, les bras fléchis, et la tête tournée en arrière signale des individus lascifs ou sexuellement déviants " (Klein 2001 : 208-209). Ce n'est alors pas la négation d'un point de vue grammatical qui est encodée, comme dans les cas que nous avons décrits plus haut, mais la négativité qualifiant l'attitude de la vie qui se répercute sur la gestuelle, et qui rapproche la figure du diable représentée dans quelques testériens de la manière de signifier le mal dans les manuscrits précolombiens.

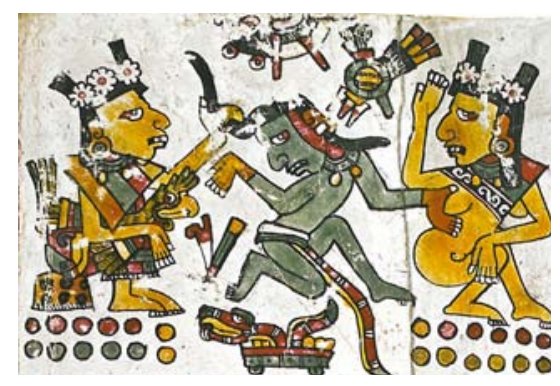

Négativité exprimée par la tête tournée (codex Borgia, "Mari adultère pris sur le fait " [Klein, 2001 : 210]).
-
12. Mis à part quelques exemplaires rédigés en boustrophédon, la lecture des testériens s'effectue en général de gauche à droite. D'ordinaire, les visages de profil sont tournés dans le même sens que celui de la lecture et les images à la forme négative se trouvent donc en général tournées vers la gauche.
13. Dans le décalogue, nous n'avons pu observer ce procédé que dans les exemplaires 809 et 830. Dans les 807, 808 et 813 , la négation y est exprimée non pas en inversant l'orientation des visages mais en croisant les doigts des personnages. Dans les autres testériens, c'est à la mémoire du lecteur qu'est confié le soin de formuler la phrase à la forme négative. 


\section{-}

14. Le mot «nom » n'est pas non plus précisé dans le testérien 813 (groupe Egerton) mais dans son cas, la présence de nombreux phonogrammes et de gloses en nahuatl indiquent plutôt que le texte pictographique est très résumé.

15. Puisque la traduction en n'importe quelle langue indienne comporte a priori l'expression «son nom est », il est à ce stade impossible de déterminer de quelle langue il s'agit précisément. On peut en revanche certifier que ces catéchismes n'étaient ni écrits ni lus en castillan.

\section{L'inscription variable dans les langues indigènes: syntaxe et phonogrammes}

Les logogrammes et déterminatifs que nous venons de décrire peuvent être compris par des locuteurs de n'importe quelle langue, et on peut imaginer qu'ils ont pu servir tant à l'instruction des enfants espagnols qu'à celle des anciens Mexicas parlant nahuatl par exemple. En comparant différentes versions des textes, en castillan ou en langues indigènes, à des ensembles logographiques plutôt qu'à des pictographies isolées, et même s'il s'agit de séquences dans lesquelles seuls des logogrammes ont été employés, le mot à mot indique parfois qu'une langue en particulier a été privilégiée lors de l'encodage. Certaines séquences d'images correspondent en effet à la syntaxe des versions indigènes du catéchisme et fournissent de la sorte des indices supplémentaires concernant leur lecture, les lecteurs auxquels elles étaient destinées, et les langues maîtrisées par leurs auteurs.

Par exemple, en espagnol, dans la liste des sacrements, on dit : El primero es el Bautismo ("Le premier est le baptême»). En revanche, dans une langue indigène comme le nahuatl, on dira Inic centetl yehuatl yn nequaatequiliztli in itoca bautismo : "En premier [c'est] lui, l'acte de verser de l'eau sur la tête, son nom est baptême." Entre le néologisme créé pour traduire le mot «baptême " (nequaatequiliztli) et l'emprunt au castillan (bautismo), l'expression in itoca, "son nom [est] » est une formulation propre à la version indigène : elle ne trouverait guère de justification dans les textes en castillan. Or, cette variation se répercute parfois au sein des phrases-images testériennes, fournissant ainsi la preuve d'une écriture et d'une lecture en langue indigène. Dans la plupart des catéchismes (parfois strictement logographiques), les phrases contiennent en effet une image - déjà rencontrée dans le Notre Père - qui a servi à écrire le mot «nom » (pour la phrase «son nom [est]»). Quant au terme « baptême», il a généralement été écrit au moyen du symbole de la colombe du Saint-Esprit.
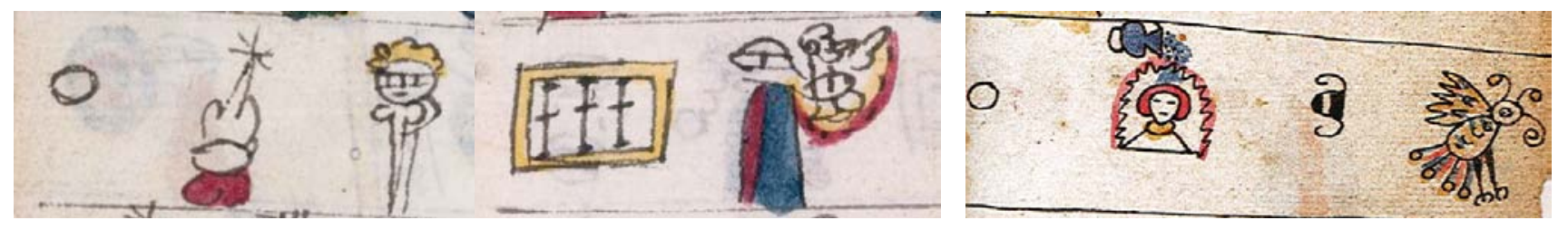

Versions indigènes : «[En] premier [c'est] lui, “l'acte de verser de l'eau sur la tête”, [son] nom [est] baptême.» $(806,814)$
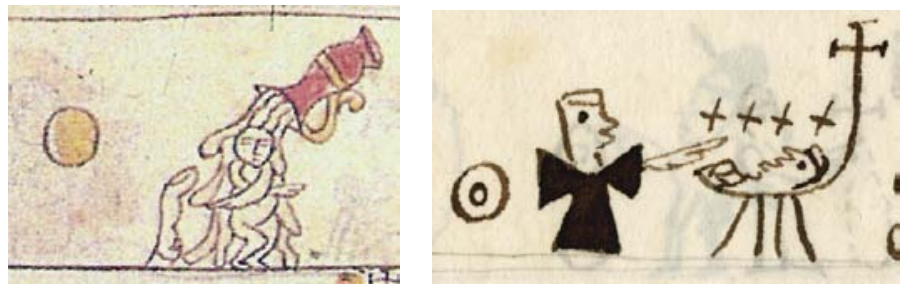

Versions castillanes : «[Le] premier [est le] Baptême.» (809 et 823)

Puisque seuls les 807 et 809 (groupe Sahagún) et les testériens du groupe Icazbalceta (dont le 823 ) ne précisent pas le mot «nom ${ }^{14}$ », la phrase y a en conséquence été notée en castillan, alors que dans tous les autres exemplaires, il s'agit de versions indigènes, par exemple en langue nahuatl ou otomi ${ }^{15}$. 
Certaines séquences d'images ne peuvent correspondre à des versions castillanes du catéchisme et sont en réalité des traductions iconographiques de textes préalablement traduits dans les langues autochtones. Or, ignorant ces disparités, de nombreux spécialistes affirment que les images testériennes ont été créées pour palier aux difficultés linguistiques et peuvent donc se lire dans toutes les langues, les traduisant donc en anglais (Dean 1989), en allemand (Anders 1988) ou bien en castillan (Resines 1992, 2007), voire les comparant à une version latine du catéchisme (Bernand 2009).

Comme on l'a vu, cette façon d'appréhender les images ne permet pas de toutes les comprendre. De plus, hormis les exemplaires des groupes Sahagún et Icazbalceta, les testériens ont été écrits et devaient être lus en nahuatl, langue prioritaire de l'évangélisation de la Nouvelle-Espagne : "Les religieux travaillèrent avec une telle ardeur à sa diffusion qu'en 1584, de Zacatecas au Nicaragua, on trouvait partout des Indiens qui la connaissaient." (Ricard 1933: 68) Ainsi, comme l'ont fait Galarza et Monod (1980) ou Berger (2002) lorsqu'ils ont étudié le 813, ou bien encore Cortés Castellanos (1987) dans son étude du 806, nous avons comparé les différents testériens aux traductions du catéchisme en langue nahuatl, ce qui permet de livrer quelques hypothèses relatives à la fonction phonographique de certaines images.

Afin de réduire le nombre des ambiguïtés pouvant survenir lors de la lecture, certaines écritures privilégiant le recours à l'image ont en effet développé un sous-système : celui des phonogrammes. Ayant pour origine un processus d'emprunt phonétique, ce sont des signes conventionnels, aisément identifiables ou plus abstraits, qui ne sont utilisés que pour les phonèmes que leur lecture invite à prononcer. Ils servent donc à écrire d'autres mots, ou parties de mots, avec lesquels les images n'entretiennent aucun rapport sémantique mais se trouvent répondre à la même structure phonémique. Bien que le terme "rébus" désigne parfois une combinaison de logogrammes et de phonogrammes, l'emploi que nous en faisons ne se réfère qu'à ce procédé plutôt ludique, effectué grâce à l'homophonie.

L'homophonie ne fonctionne en général que dans une langue et la présence de ces signes permet donc d'établir et d'affirmer précisément en quelle langue étaient lues les images.

Dans le 813, l'image de la «main» (ma-itl en nahuatl) a servi à écrire les phonèmes $/ \mathrm{ma} /$. Ils sont en effet identiques à ceux du lexème ma qui marque en nahuatl un verbe à l'impératif (Galarza et Monod 1980). Dans le Notre Père, toujours au moyen d'une formule impérative, le croyant prie pour "que la volonté de Dieu soit faite", tandis qu'on supplie Dieu le Père dans les phrases "faites que nos péchés soient pardonnés", "que vous ne nous laissiez pas tomber dans la tentation" et "que vous nous délivriez du mal». Quasiment à chaque "que...", ma en nahuatl, une main-phonogramme a été représentée. Dans ce cas, tous les sons ont été écrits mais il arrive que l'écriture phonographique d'un mot lui fasse perdre certains phonèmes. Les phonèmes manquants doivent alors être restitués durant la phase de lecture. Cette élision du son peut être mise en évidence dans l'exemple du logogramme momoztli, "autel», employé comme phonogramme pour l'écriture du mot momoztlae, "quotidien" (Aubin 1885: 29; Galarza et Monod 1980: 79). On le trouve dans les exemplaires 806 et 813 (autels pyramidaux représentés en coupe). Dans les 810 et 814 , les autels sont circulaires : ils ont été représentés vus de haut et sont entourés par des traces de pas ${ }^{16}$.
8

16. II est possible que les traces de pas indiquent de façon redondante le son $/$ tla/. On trouve en effet une trace de pas similaire, lue tlalli dans le toponyme Tlaltizapan (codex Mendoza, planche 54, fig. 2). On notera le caractère non systématique de l'écriture testérienne puisque dans le 809 , le logogramme «soleil » est répété trois fois pour écrire «quotidien ». 
Dans ces deux exemples (maitl pour /ma/ et momoztli pour /momostl/), la correspondance entre les sons est parfaite ou presque. Les images fournissent quasiment la totalité des sons des mots qui devaient être lus.
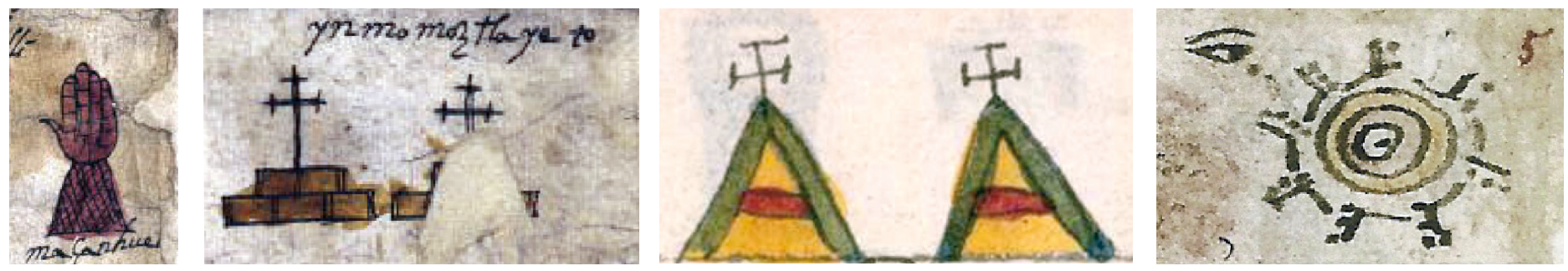

La marque de l'impératif «ma » dans le testérien 813 et de l'adverbe « quotidien » dans les 813, 806, 810.

D'autres rébus fonctionnent plutôt comme des déclencheurs. Ces phonogrammes "prospectifs » aident à la remémoration du mot mais n'en fournissent que les phonèmes initiaux. Dans le 810, par exemple, un œil ponctue le texte pictographique tout au long du catéchisme. En supposant que cet œil devait être lu en nahuatl, ixtli, seul son phonème initial (/i/) devait être conservé par le lecteur. Il lui ajoutait ensuite le phonème $/ \mathrm{n} /$ pour prononcer finalement in, particule nahuatl signifiant «le, la, les», employée aussi pour le pronom relatif « qui ».

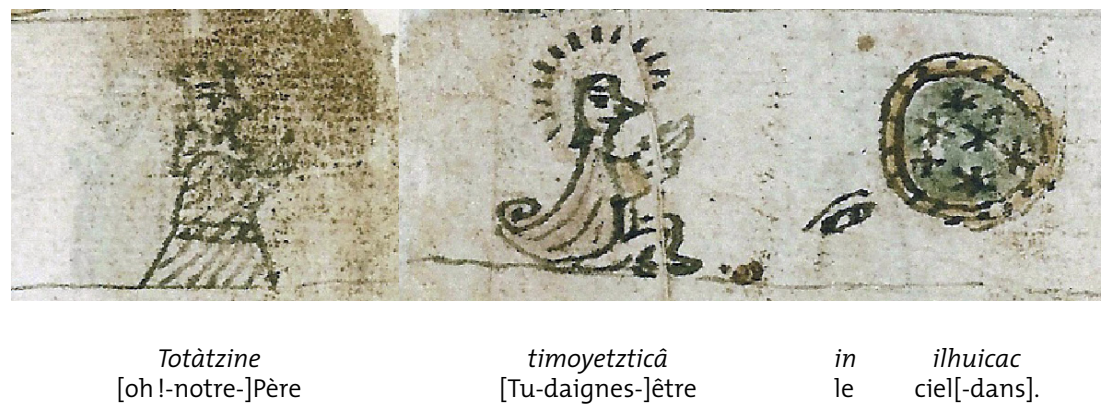

On retrouve ce procédé dans le système égyptien, où les signes appelés unilitères peuvent être formés par acrophonie, c'est-à-dire en utilisant un signe pour la valeur initiale du mot ou du son qu'il note. Dans notre exemple, on remarque en outre que les images correspondent parfaitement à la syntaxe de langue nahuatl. Aux mots agglutinés comme ilhuica-c, composé avec la racine ilhuica, "ciel » et le suffixe locatif - $c$, " dans ", une seule image correspond. Un scribe français aurait peut-être cherché des images pour écrire les trois mots «dans », «le» et «ciel».

Enfin, d'autres phonogrammes fonctionnent de façon rétrospective. C'est le cas, par exemple, de l'adverbe positionnel ihuicpa, "contre", employé dans la phrase "délivre-nous contre tout mal", ma xitechmomaquixtili in ihuicpa in ixquich àmo cualli. Alors que celui-ci n'a pas toujours été écrit, il a été noté dans le 808 par le truchement d'une bannière. Cette image, dont on connaît l'emploi dans les manuscrits mexicas du type codex Mendoza ou Matrícula de Tributos, est usuelle pour l'écriture des noms de lieux. La bannière, pantli en nahuatl, 
servait en effet à noter les phonèmes /pa/ ou /pan/, terminaison locative de nombreux toponymes. Tochpan par exemple, "lieu des lapins", a été écrit dans le codex Mendoza au moyen d'un lapin sur le dos duquel a été représentée une bannière. L'emploi s'avère donc différent dans les testériens, où la bannière note un adverbe. Le principe n'est pas non plus tout à fait le même puisque, à la différence du toponyme complété par l'image du logogramme «lapin " (toch-tli), seule la terminaison du mot (ihuicpa) a été enregistrée dans les testériens. Dans le 813, la bannière a aussi servi à écrire les adverbes topampa ("pour nous»), ipampa (" pour lui »), pampa ("parce que») et zatepan («ensuite») : en lisant le son /pa/, le lecteur devait effectuer une inférence pour retrouver la totalité du mot.

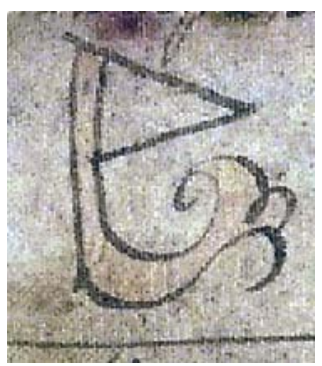

a
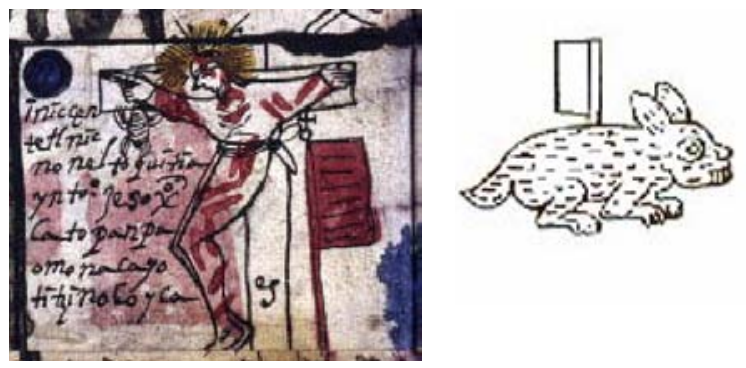

$\mathrm{b}$
90

17. II s'agit de la lexicalisation de la forme verbale iuh (au présent] ou ihui (au prétérit) sous la forme d'un adverbe «ainsi, comme » (Karttunen 1992 : 101).

18. L'ahuizot/ est une créature légendaire comparable à un castor ou à une loutre, décrite par fray Bernardino de Sahagún [1979, liv. 11) comme ayant des oreilles pointues et une longue queue se terminant par une sorte de main, grâce à laquelle le monstre attirait les hommes dans les profondeurs aquatiques afin de les noyer.

a. ihuicpa, "contre" (808); b. omonacayotitzinoco topampa, «il s'est fait homme pour nous" (813); c. Tochpan, «lieu des lapins" (codex Mendoza).

La suite phonémique /iw/ ou /iwi/ est d'ailleurs très intéressante en termes de phonogrammes. Les phonèmes sont contenus dans un lexème que l'on écrit conventionnellement iuh(i) ${ }^{17}$, et qui a servi à traduire "comme, ainsi [que] ». On le retrouve dans deux phrases du Notre Père "que Ta volonté se fasse sur la terre comme au ciel " et "pardonne-nous comme nous pardonnons". Dans plusieurs testériens (809, 810, 823, 824, 830 et 832), à l'endroit où le terme " ainsi » doit normalement être énoncé, des scribes ont représenté un petit animal. Sans qu'en soit pour autant proposé une lecture, sa présence a été remarquée par de nombreux auteurs qui l'ont décrit comme une «souris» ou un «rat " (Orozco y Berra 1877), un "tatou " (Dean 1989), un "cochon" (Resines 2007), un "raton laveur" (Bonilla 2004 : 201) ou bien un «ocelot» (Bernand 2009: 35).
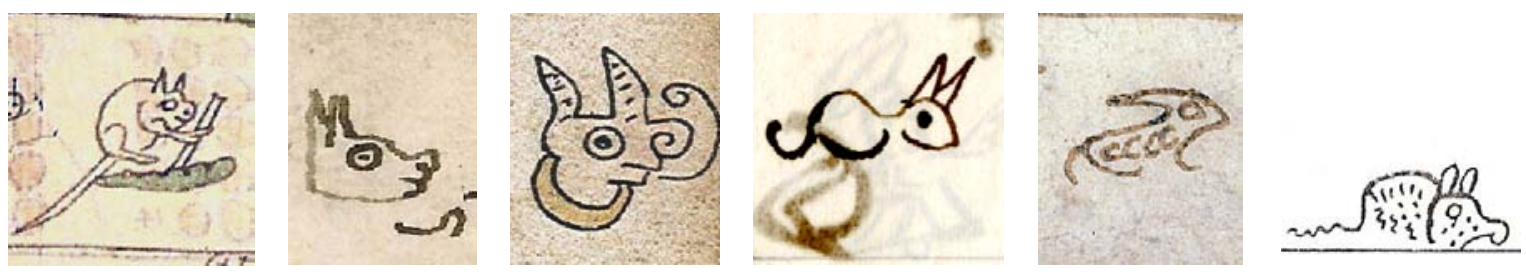

La particule « ainsi que » dans les testériens 809, 810, 814, 823, 824 et 830 .

Nous pensons qu'il s'agit en réalité d'un ahuizotl ${ }^{18}$ (/awisotl/) qui, employé pour des raisons phonographiques, aurait servi à écrire les sons /wi/ contenus dans iuh(i) (prononcé /iw(i)/), « ainsi ». 


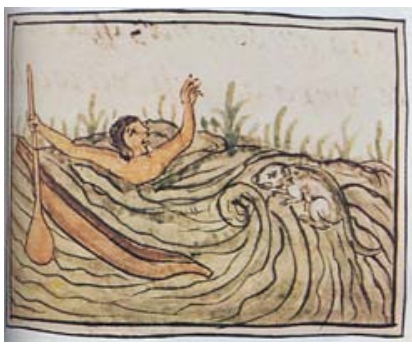

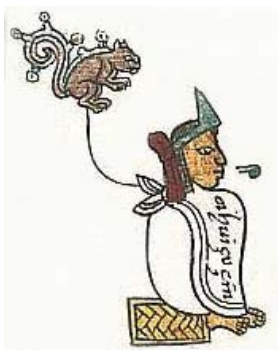

b

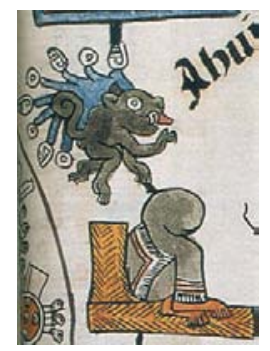

C

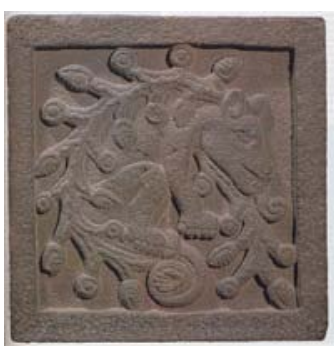

$\mathrm{d}$

Différentes représentations d'un ahuizotl : a. codex de Florence,

b et c. glyphe anthroponymique de l'empereur Ahuizotl relié à sa représentation ou à celle de son paquet funéraire (codex Mendoza et codex Telleriano-Remensis),

d. bas-relief en provenance de Tepoztlan.

On trouve également cette locution dans la formule "ainsi soit-il», ma iuh(i) mochihua (littéralement "que ainsi [cela] se fasse»), qui a été écrite à l'aide de deux ensembles phonographiques différents. Dans le premier, l'ahuizotl est suivi d'une "fleur", parfois très stylisée, lue xochitl. Celle-ci est utilisée pour noter les sons /otch/ contenus dans le verbe "faire» ici conjugué à la forme réfléchie, mochihua. Dans le deuxième (813), plutôt que d'insister sur le son /wi/, le scribe a préféré mettre l'accent sur le son $/ \mathrm{ma} /$ du début de la formule. Il a donc placé une "main", maitl, suivie d'une "fleur", xochitl, toujours pour le son /otch/. Pour écrire /ma/ /iwi//motchiwa/, nous constatons donc l'usage de plusieurs solutions scripturales qui favorisent deux des trois sons suivants : /ma/, /wi/ et /otch/19.
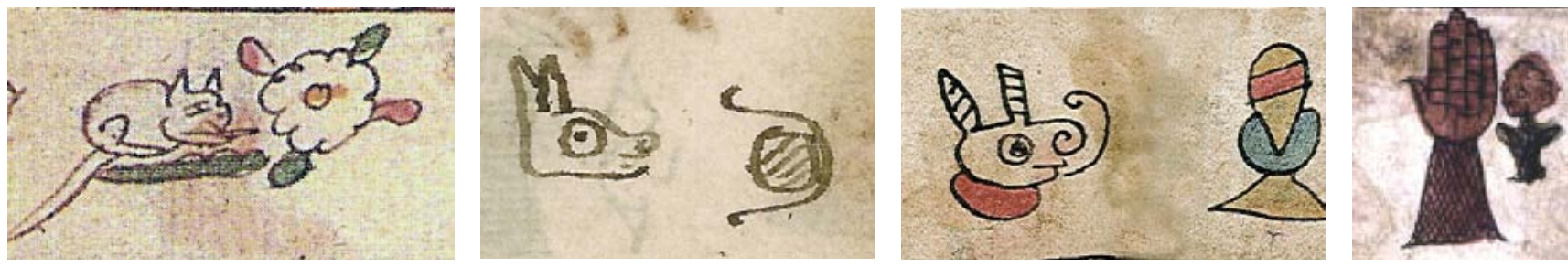

«Ainsi soit-il » dans les testériens 809, 810, 814 (ahuizotl + fleur) et 813 (main + fleur).

Cette diversité sémiotique est sans doute liée à des contraintes linguistiques. Alors que des phonogrammes ont parfois été employés, ceux-ci ne pouvaient être lus qu'en nahuatl. Afin d'évangéliser d'autres régions, et donc d'exporter la méthode, les scribes auraient donc expurgé le catéchisme initial (le "prototype »?) de tous les phonogrammes qu'il contenait initialement. L'inverse est aussi possible : pour rendre son catéchisme plus attractif, plus inventif, plus adapté à la langue nahuatl, un scribe a tout à fait pu y insérer des images auparavant inexistantes. Désireux d'améliorer ce qu'il copiait, le scribe pouvait proposer des phonogrammes à la place de logogrammes peu clairs, montrer sa maitrise de la langue, de l'écriture traditionnelle, ou bien du répertoire iconographique propre aux religieux : il jouissait d'une relative liberté et pouvait déployer une créativité personnelle ou bien se faire l'héritier d'une tradition scripturale et iconographique particulière. En outre, le fait de copier un catéchisme a également pu engen- 
drer des erreurs, un copiste omettant d'ôter (ou inversement de recopier) certains phonogrammes. Enfin, la fonction de certains phonogrammes aurait pu glisser, ceux-ci acquérant avec le temps le statut de signe conventionnel.
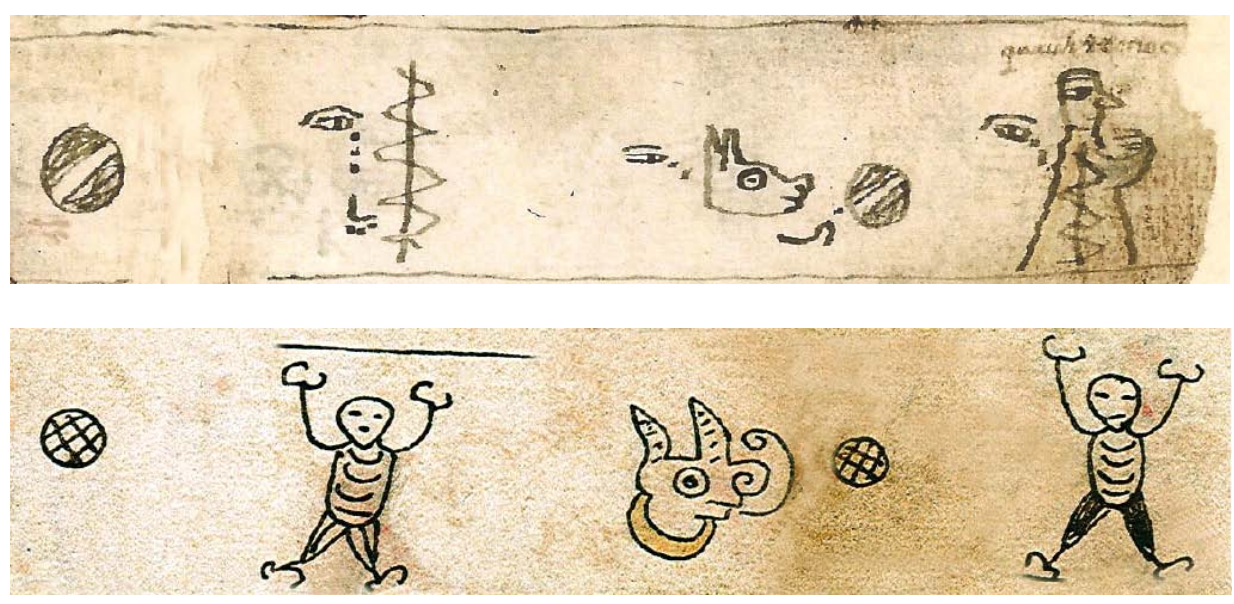

«[... et] pardonnez[-nous nos] offenses comme [nous] pardonnons [à ceux qui nous ont] offensés ».

La comparaison du 810 et du 814 (sans doute élaboré à partir du premier ${ }^{20}$ ) fournit un exemple de ces disparités. Dans la phrase "pardonne-nous nos offenses comme nous pardonnons ceux qui nous ont offensés" (en nahuatl: ma xitechmopopolhuili in totlàtlacol in iuh tiquimpopolhuia in techtlatlacalhuia), on constate la disparition des trois phonogrammes «œil» (pour /in/) et de la petite «trace de pas » reliée au premier d'entre eux (pour /tla/ $/{ }^{21}$ ?). En outre, le signe du "péché », logogramme plutôt abstrait constitué par une ligne verticale traversée par une autre ligne ondulante, a été remplacé par un être griffu personnifiant la faute. Le peintre du 814 aurait pu effectuer de tels changements pour faciliter la compréhension et favoriser une diffusion plus importante du cahier auprès de populations locutrices de langues différentes du nahuatl. Mais le scribe a pourtant conservé l'ahuizotl. Or, l'ahuizotl est également présent dans des catéchismes du groupe Icazbalceta (comme les 823 et 832 ) qui, selon nous, pouvaient être lus dans diverses langues et en priorité en espagnol.

On peut donc penser que la lecture de ce signe a fini par prendre, avec le temps, une certaine indépendance vis-à-vis du son émis lors de sa désignation en nahuatl. Passant de la fonction initiale de phonogramme à celle de logogramme, l'image se serait convertie en un signe conventionnel permettant d'écrire le mot «comme, ainsi » d'une manière compréhensible par tous.

L'application systématique de notre méthode a donc permis d'établir des groupes distincts de testériens. Il y a ceux qui sont très résumés (le 817), ceux qui sont condensés mais qui présentent certaines subtilités de la langue nahuatl (le 813), et ceux dont le texte est écrit quasiment mot à mot. Parmi ces derniers, il est possible quse certains aient été écrits/lus en espagnol (groupe Sahagún [807 et 809] et groupe Icazbalceta [ 823 et 832 , entre autres]) alors que d'autres l'étaient assurément en langues indigènes (groupe Gante [dont le 806], groupe Egerton [813], groupe Tulane Brown [824 et 827] et groupe des isolés [830, 808, 810, 814 et 844$]$ ). Nous avons aussi pu mettre en relief différentes propriétés des images
- -

20. Si l'on en croit la date inscrite dans le texte figurant à l'intérieur du testérien 810 , celui-ci a été réalisé en 1591. Sans éléments tangibles permettant de l'affirmer, on situe l'élaboration du 814 au cours du xvIl ${ }^{\mathrm{e}}$ siècle (Normann 1985 : 427) ou bien entre la fin du $x v I^{e}$ et le début du xvII ${ }^{e}$ (Bernand 2009 : 23-24).

21. II s'agit certainement aussi d'un phonogramme correspondant aux phonèmes /tla/ contenus dans tlàtlacolli, offense ; voir n. 16. 
testériennes. Dans certains documents, l'écriture semble être purement logographique (groupe Gante et groupe Icazbalceta). Les exemplaires du groupe Icazbalceta, nettement plus influencés par l'iconographie européenne que mexica, pourraient d'ailleurs être comparés aux catéchismes micmacs utilisés et élaborés par les récollets et utilisés à partir du xviI ${ }^{\mathrm{e}}$ siècle en Nouvelle-France. Bien que l'aspect figuratif de l'iconographie testérienne perdure au sein de ce groupe de manuscrits, l'emploi exclusif de logogrammes peut évoquer les signes abstraits et arbitraires des logogrammes micmacs, mêlés à des lettres ou à des caractères usités à l'époque en chimie (Déléage 2009 : 95). Dans d'autres catéchismes, et notamment les $808,809,810,813$ et 814, il s'agit d'un système mixte, ou logo-phonographique, combinant des logogrammes à des phonogrammes ${ }^{22}$.

\section{Des images et leurs usages : entre lecture et déclenchement mémoriel}

La variété des solutions pictographiques que nous venons de décrire reflète certainement des conceptions divergentes vis-à-vis de l'écrit. En effet, certains, accordant peut-être moins de confiance à la mémoire individuelle du lecteur, choisissent d'encoder beaucoup plus de mots que d'autres. En conséquence, et en particulier en ce qui concerne les rébus, comment ces procédés fonctionnent-ils dans la pratique? Puisque la question des auteurs reste sans réponse, peut-on comparer les rébus que nous venons de décrire au système pictographique aztèque ou sont-ils assimilables aux rébus européens? En outre, l'application de catégories scripturales strictes telles que logogramme, phonogramme ou déterminatif conduit à isoler les images les unes des autres alors que celles-ci fonctionnent ensemble, leur agencement dans la page participant lui aussi à la remémoration du texte pour le lecteur. En effet, les testériens ont été écrits pour être lus à voix haute. Se pose alors la question de la fonction de l'image dans le stade de lecture, du passage de l'image écrite à celui de l'oralité ou plutôt de la literacy, définie comme la capacité à relier le langage à l'écrit (Houston 1994: 27).

Les phonogrammes représentent des signes qu'il est nécessaire de prononcer afin que ceux-ci fonctionnent. On a vu que les images endossant cette fonction ne restituent pas la totalité des sons contenus dans le mot qui devait être prononcé. Il existe des rébus complets ou quasi complets, d'autres prospectifs ou bien rétrospectifs : parfois, seuls les phonèmes contenus au milieu ou bien à la fin d'un mot sont transmis par l'image. C'est une caractéristique propre aux testériens, révélant à nouveau l'originalité de la méthode, mais soulevant également des interrogations au sujet de leur efficacité.

En effet, dans les codices dits traditionnels, la majorité des phonogrammes ont aussi servi à écrire des mots plutôt abstraits, comme par exemple les suffixes locatifs ("sous", "dans", "près de", «au-dessus") ou bien des diminutifs et des augmentatifs («petit», "gros»), ainsi que des suffixes révérenciels. Mais ils étaient associés en général à des logogrammes, et si la fin des mots n'était pas systématiquement marquée, le début d'un mot était en revanche toujours

22. Le caractère mixte du 809 reste encore à démontrer. Nous n'avons pu présenter que de rares hypothèses concernant l'usage de rébus dans ce manuscrit. Bien qu'il contienne l'ahuizotl, celui-ci a pu acquérir avec l'usage la fonction de logogramme. encodé : «Les phonèmes indiqués par les signes d'un composé ne doivent pas forcément être contigus [...], en revanche, ils doivent toujours représenter au moins la première syllabe du mot. " (Lacadena $2008: 14$ )

Le fonctionnement des rébus, créés dès le $\mathrm{xvI}^{\mathrm{e}}$ siècle en Europe, s'apparente-til mieux à celui employé dans les testériens? Différents systèmes coexistaient, jouant tant sur les mots que sur l'homophonie. Concernant les rébus de la Renais- 
sance, Céard et Margolin (1986) ont publié une série d'images issues de livres d'Heures exécutés par Guillaume Godard au début $x^{\mathrm{e}}$ siècle et transcrivant le texte d'une prière à la Vierge Marie entièrement écrite au moyen d'un système mixte ${ }^{23}$. Cet assemblage d'images fonctionne a priori de la même manière que les testériens. On retrouve en effet certaines caractéristiques telles que la combinaison de logogrammes ("Marie priant", "Jésus en croix", "cœur", "Dieu») et de phonogrammes (la pièce de monnaie appelée «salut" pour les sons /salu/, une "scie" pour/si/, un "mont " pour /mon/, un «loup" pour /lou/, un «ange" pour /ange/) dont certains jouent sur le principe de l'acrophonie (un «os " pour le son /o/) ou ne retiennent que la première suite phonémique consonne-voyelle (un «sac» pour /sa/, un «dix» écrit en chiffre romain pour le son /di/).

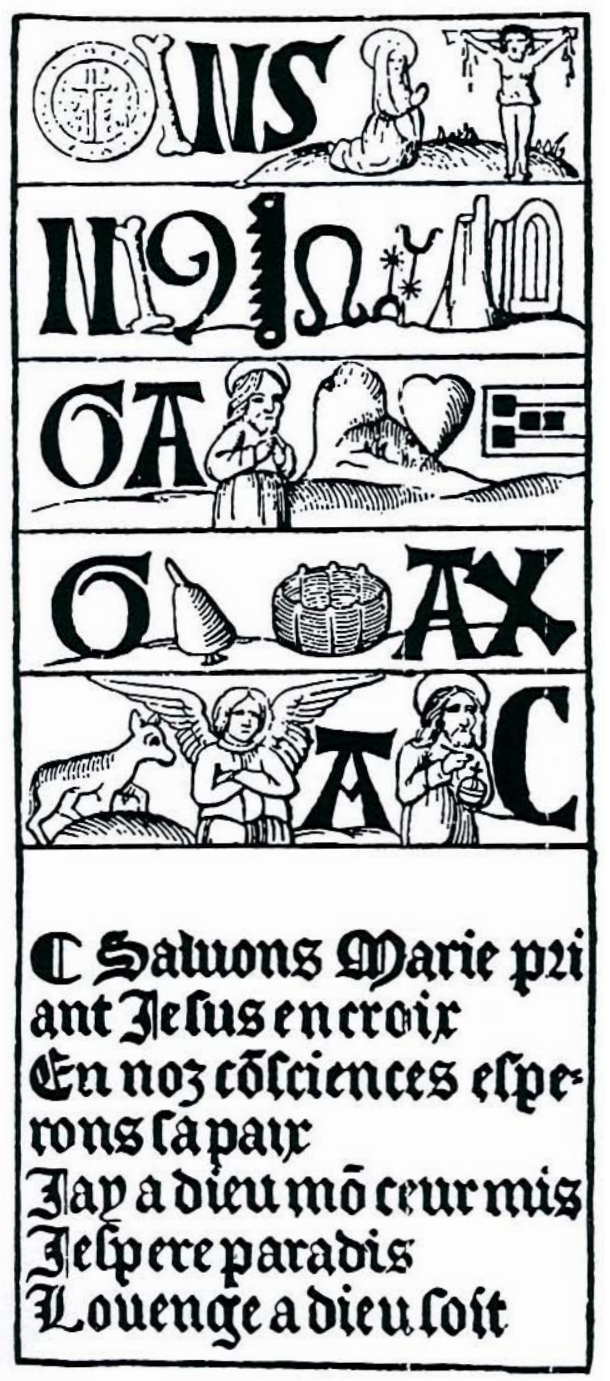

1. Saluons Marie priant Jesus en croix salut/os/N/S

2. En noz co[n]sciences esperons sa paix N/os/con(note tironienne)/scie/anse/ é[s]peron/sac/paix 3. J'ay a Dieu mon cœur mis $\mathrm{G} / \mathrm{A} / \mathrm{Dieu} / \mathrm{mont} / \mathrm{cœu} / \mathrm{mi}$

4. J'espere paradis $\mathrm{G} / \mathrm{poire} / \mathrm{parc} / \mathrm{A} / \mathrm{dix}$

5. Louenge a Dieu soit

loup/ange/A/Dieu/C(soit)

Guillaume Godard, Rébus en forme de prière, d'après les Heures de Paris, 1513-1523, in Céard et Margolin $1986: 171$.

Néanmoins, le procédé de ce rébus français diffère sur plusieurs points avec la technique testérienne. En premier lieu, le nom des lettres doit seulement être prononcé : la solution n'exige aucun effort de la part du lecteur. En revanche, lorsque les auteurs des testériens utilisent les lettres de l'alphabet, c'est pour donner l'initiale des emprunts (on trouve par exemple un B majuscule dans le 808 pour bautismo). Le lecteur devait donc compléter l'information par lui-même. En outre, les phonogrammes testériens sont fondés sur une quasi-homophonie : il faut d'abord comprendre qu'il ne s'agit pas d'un logogramme, puis ôter au mot initial certains de ses composants phonétiques et grammaticaux (le début du mot ou le suffixe absolu des substantifs par exemple). Ensuite, le lecteur doit reconstituer le mot qui prend effectivement place dans la prière. Mais le travail de lecture ne s'arrête pas là : il faut également, on l'a vu, reconstituer les phrases entières et se souvenir des mots non précisés par le scribe. Ces éléments réunis montrent les
- 9

23. Ces auteurs situent dans les années 1470-1510 l'intensification de la production de rébus dans l'espace français et francobourguignon, ainsi que dans l'espace italien des faiseurs d'imprese (devises) relatives à la rhétorique amoureuse qui fleurissaient dans les mêmes décennies (Céard et Margolin 1986 : 165). 
- 0

24. Les lettres $\mathrm{N}$ et $\mathrm{S}$ ont été ajoutées dans le livre d'Heures pour des questions orthographiques après le «salut » et l'《OS $\gg$ (pour «salu-o-n-s»). En revanche, dans un testérien, les règles orthographiques n'influent jamais sur le choix des images. importantes différences avec la prière en rébus français du début du $\mathrm{xvI}^{\mathrm{e}}$ siècle, dans laquelle chaque son de la totalité des mots de l'oraison a été enregistré24.

La technique testérienne est donc, de ce point de vue, originale. Elle ne s'apparente totalement ni à celle des manuscrits traditionnels mexicains élaborés depuis l'époque précolombienne, ni à celle des prières logo-phonographiques créées dans l'Ancien Monde. Décrire le fonctionnement de l'image phonographique ne permet donc pas d'identifier les auteurs des testériens, mais permet en revanche de formuler une autre interrogation, concernant le rôle de l'image lors du stade de la lecture qui n'est pas, on a pu le constater, totalement guidée - ni tous les mots, ni tous les sons ne sont indiqués. À l'exception de certains phonogrammes ayant probablement acquis avec l'usage le statut de signes conventionnels connus et déchiffrables par tous, les rébus testériens ne peuvent être compris que si le texte a été préalablement mémorisé. Puisqu'ils ne permettent qu'une remémoration, quel est alors le rôle de l'image en tant que déclencheur de mémoire?

Il est possible que la place occupée par les images au sein de la page ait été pertinente, jouant en faveur d'une remémoration plus efficace. Selon Carruthers (2002 : 207), «la marge est, dans la page des manuscrits médiévaux, l’endroit par excellence où le lecteur peut s'adonner au travail de la mémoire et lui donner toute latitude». Si les testériens ne possèdent pas de marges, la récurrence ou la place des images ont certainement été pensées dans le but de provoquer un impact sur la mémoire du lecteur.

Il semble par exemple que certaines images ne sont efficaces que parce qu'elles scandent le texte testérien, participant de la sorte à l'art de la psalmodie. En effet, à la différence des pictographies amérindiennes où la "formule-base " «n'est jamais traduite par l'image pictographique [et dont] l'apprentissage est confié à la seule mnémotechnique orale » (Severi 2007 : 169), les catéchismes testériens reproduisent, en même temps que les variations, l'échafaudage narratif, c'est-à-dire la charpente du texte. Afin que le catéchumène les lise, les mémorise, et les répète à l'oral, les mots redondants, écrits au moyen de solutions, certes, disparates, sont généralement précisés. En effet, les textes testériens, issus du catéchisme, sont en général constitués de listes fixes et numérotées. Tous les nombres se retrouvent dans les testériens et ordonnent les séquences d'images. De même, on retrouve parfois la tournure des propositions, comme la forme négative dans une partie des dix commandements : "Tu ne tueras point. Tu ne forniqueras point. Tu ne voleras point, etc. ", ou bien encore l'expression "bienheureux qui» dans la liste des huit béatitudes. Ce sont des éléments iconiques récurrents qui, à la façon des mots répétés dans le texte, rendent l'ensemble efficace et participent de la sorte à la remémoration.

C'est aussi le cas des structures verbales qui sont répétées tout au long de ces listes. Par exemple, le verbe "croire » est répété quatorze fois dans la liste des quatorze articles de la Foi : «En premier, je crois que Dieu est Père. En deuxième, je crois que Dieu est Fils. En troisième, je crois que Dieu est Saint-Esprit, etc.» L'expression " je crois » a été écrite dans le 817 sous la forme d'un rectangle parsemé de petits traits, vraisemblable référence à l'écrit, sorte de catéchisme miniature à l'intérieur même du catéchisme. Dans le 813, en revanche, un phonogramme a été employé par le peintre. Le verbe a été écrit au moyen d'une araignée, tocatl en nahuatl dont la prononciation fournit les phonèmes /toc/ contenus dans la formule «je crois», nitlaneltoca (ou nicnoneltoquitia à la forme révérencielle). Si en 
effet le jeu sur l'homophonie apparaît judicieux, l'image décontenance autant que celle de l'ahuizotl utilisé pour exprimer « ainsi soit-il ». Elles jouent alors peut-être un rôle supplémentaire : placées à des endroits stratégiques et prenant par surprise les lecteurs, ces images décalées par rapport au propos général des textes serviraient à fixer dans la mémoire les textes catholiques laborieusement étudiés.

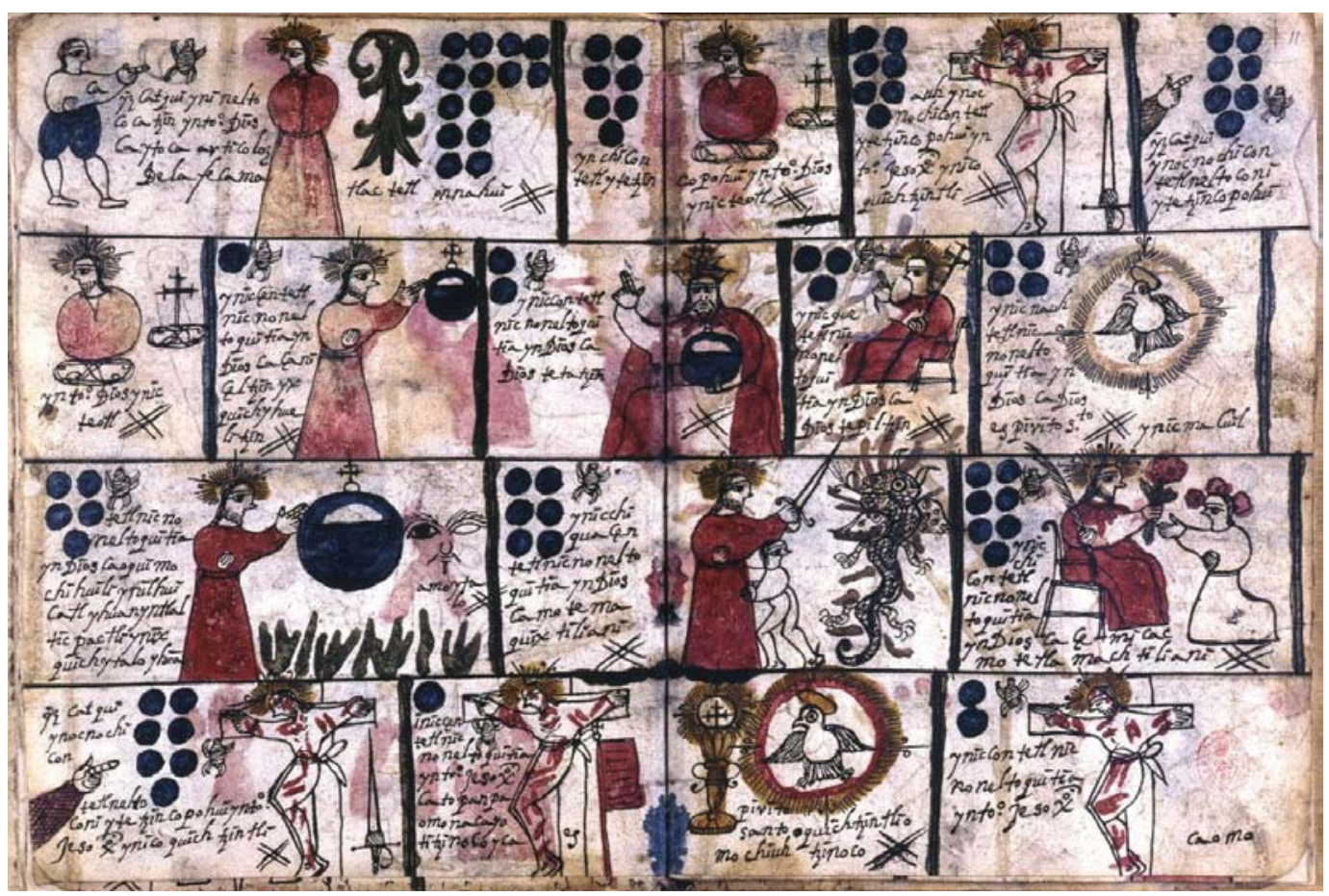

Les articles de la Foi (813, détail : fol. 10v11r) : à côté de chaque groupe de cercles-unités, les phonèmes /toc/ contenus dans la formule nitlaneltoca, "je crois», ont été notés au moyen d'une araignée, tocaitl. On doit lire ici : «en premier, je crois... », «en deuxième, je crois... », etc.

Cette plurifonctionnalité de l'image s'oppose aux considérations formulées au sujet de l'image chrétienne, longtemps perçue en Europe comme «Bible des illettrés ", un substitut du texte déprécié. Pourtant, bien que le pape Grégoire le Grand insistait en 600 sur la fonction instructive de l'image, il considérait à la fois deux autres fonctions des images : «Apprendre ce n'est pas seulement découvrir mais aussi se remémorer, de sorte que l'image a pour rôle d'entretenir la pensée des choses saintes; en outre, elle peut émouvoir l'esprit, susciter un sentiment de componction qui permet de s'élever vers l'adoration de Dieu. " (Baschet 1996 : 8) Certaines images testériennes joueraient en cela le même rôle que les images médiévales. Les grotesques, par exemple, sont des monstres effrayants qui servent non seulement à éveiller la crainte et l'angoisse, mais conduisent en même temps à amorcer la méditation. Ce sont des images «amusantes, propres à réjouir la cellula deliciarum de la mémoire, à sortir l'esprit de sa torpeur " (Carruthers 2002 : 210). Le lecteur ne doit pas en conséquence rester passif face au texte, car l'enjeu est avant tout de mémoriser par cœur, mais bien également de «suivre le vrai", de croire et de méditer durant l'acte de prière. Ces animaux, à l'instar de certains éléments répétitifs, tels que les nombres ou les syntagmes récurrents ("son nom est ", "bienheureux celui qui ", " je crois que ») endosseraient en conséquence la fonction de «rythmeurs » et au-delà, d'embrayeurs mémoriels. 
Ainsi, qu'elle soit européenne ou indigène, cette technique scripturale mobilise toute une gamme de fonctions imputées aux images en général. L'une d'entre elles est certainement celle d'animer le texte, de lui donner corps par l'action de mise en voix nécessaire non seulement à la lecture mais aussi à la compréhension. Les rébus, notamment, obligent le lecteur à les nommer; il faut prononcer leur nom pour qu'ils soient compris et efficaces. En même temps, ils peuvent aider à la mémorisation, à la méditation et à la prière orale. L'emphase sur l'usage de l'image, à partir de la moitié du xvi ${ }^{\mathrm{e}}$ siècle suite aux recommandations du Concile de Trente, doit être mise en relation avec l'intérêt pour les arts de la mémoire développés en Europe à la fin du Moyen Âge tardif et à la Renaissance, notamment les travaux du franciscain Diego de Valadés sur les techniques de mémorisation, ainsi que ses tentatives d'adaptation au contexte mexicain avec un alphabet mnémonique destiné aux Indiens. Selon Boone, les longs enchaînements d'images testériennes reflètent les conceptions des frères missionnaires: "Les Nahuas [...] auraient simplement mémorisé les textes; ils pouvaient consulter une simple image peinte pour évoquer l'intégralité de la prière, mais ils n'auraient pas recherché [à représenter] une séquence mot à mot [...]. En ce sens, les peintures plus grandes illustrant les sermons et instructions des frères étaient plus proches de la tradition indigène que ne l'étaient les Testériens. " (Boone 1998 : 164) Cela dit, comme nous avons pu ici le démontrer, le corpus testérien est hétérogène. Il y a effectivement des catéchismes écrits mot à mot alors que pour d'autres, les phrases sont seulement évoquées. Or, cette variété des solutions pictographiques employées pour écrire les mêmes textes, en même temps qu'elle fournit des renseignements sur la langue que maîtrisaient obligatoirement les concepteurs des phonogrammes, donnent aussi des indices relatifs aux conceptions de leurs auteurs vis-à-vis de l'écrit et de la mémorisation. Par ailleurs, ces exemples illustrent la multiplicité des tentatives employées, avec plus ou moins de succès, dans une situation de contact où l'intention principale demeure l'évangélisation, qui passe de manière essentielle par l'apprentissage par cœur de longs textes destinés à être récités lors des sacrements les plus importants - baptême, confession et mariage.

Université de Paris Ouest-Nanterre-La Défense, Laboratoire d'ethnologie et de sociologie comparative bgaillemin@gmail.com

mots clés / keywords : Mexique // Mexico - catéchisme // catechism • écriture // writing • image // images $\cdot$ mémoire // memory.

\section{Bibliographie}

\section{ANDERs, Ferdinand}

1988 « ¿Hizo o no hizo ? Von der Aussagekraft Mexikanischer Schrift », in Maarten Jansen, Peter van der Loo, Roswitha Manning et Benedikt Hartmann (éd.), Continuity and identity in Native America. Essays in honor of Benedikt Hartmann. Leiden, Brill : 193-249.
Aubin, Joseph Marie Alexis

1885 Mémoires sur la peinture didactique et l'écriture figurative des anciens Mexicains. Paris, Imprimerie nationale.

\section{Balmaseda, María Luisa}

1989 « Códices testerianos 》, in Joaquín Galarza (éd.), Descifre de las Escrituras Mesoamericanas : 48 Congreso Internacional de Americanistas, Amsterdam, 1988. Vol. I. Oxford, BAR International : 185-215.

\section{BASCHET, Jérôme}

1996 «L'image-objet », in Jérôme Baschet et Jean-Claude Schmitt (éd.), L'Image. Fonctions et usages des images dans l'Occident médiéval. Paris, Léopard d'Or [«Cahier du Léopard d'Or 5 »] : 7-26. 
Batalia Rosado, Juan José

À paraître «Los manuscritos testerianos », in R. Martinez Garcia et X. Noguez (éd.), Códices y documentos de tradición indígena del Estado de México. Mexico, El Colegio Mexiquense.

\section{BERGER, Uta}

2002 Gebetbücher in mexikanischer Bilderschrift : europäische lkonographie im Manuskript Egerton 2898 aus der Sammlung des Britischen Museums. Münster, Lit Verlag.

\section{Bernand, Carmen}

2009 Teotl. Dieu en images dans le Mexique colonial. Paris, Fondation Martin Bodmer-PUF.

\section{Bonilla Palmeras, Jesús Javier}

2004 Entre imágenes y oraciones : un acercamiento al catecismo indígena 078 de la BN de París, Francia. Mexico, Escuela Nacional de Antropología e Historia, mémoire de master.

\section{Boone, Elizabeth Hill}

1998 [1992] « Pictorial documents and visual thinking in Postconquest Mexico », in E. H. Boone et T. Cummins (éd.), Native Traditions in the Postconquest World, A symposium at Dumbarton Oaks. Washington, Dumbarton Oaks Research Library and Collection : 149-200.

2007 Cycles of Time and Meaning in the Mexican Books of Fate. Austin, University of Texas Press.

\section{CAROCHI, Horacio}

2001 [1645] Grammar of the Mexican language with an explanation of its adverbs. Traduit et édité par James Lockhart. Stanford, Stanford University Press.

\section{CARruthers, Mary J.}

2002 Machina Memorialis. Méditation, rhétorique et fabrication des images au Moyen Âge. Paris, Gallimard.

CÉARD, Jean et MARgolin, Jean-Claude

1986 Rébus de la Renaissance. Des images qui parlent. Paris, Maisonneuve et Larose.

\section{Cortés Castellanos, Justino}

1987 Catecismo de Fray Pedro de Gante. Madrid, Ministerio de Educación y Ciencia.

\section{DeAn, Carolyn S.}

1989 « Praying with pictures: a reading of the Libro de Oraciones », Journal of Latin American Lore 15(2) : 211-273.

\section{DéLÉAge, Pierre}

2009 La Croix et les hiéroglyphes. Écriture et objets rituels chez les Amérindiens de Nouvelle-France (XVII ${ }^{e}$-xVIII siècles). Paris, Éditions rue d'Ulm-musée du quai Branly [«Asthetica»].

\section{Gaillemin, Bérénice}

2010 «À la recherche des prototypes perdus : pour une remise en contexte des catéchismes testériens 》, Cahiers d'Histoire de l'Amérique Coloniale 4 (numéro spécial «Villes et Sociétés urbaines en Amérique coloniale », Bernard Grunberg [dir.]) : 171-197.

À paraître «Les catéchismes testériens : un corpus homogène ? » in Actes du colloque «Nouveaux chrétiens, nouvelles chrétientés 》 (Paris Ouest-Nanterre-La Défense, 8-10 avril 2010). Nanterre, Presses universitaires de Nanterre.

GALARZA, Joaquín et Monod-Becouelin, Aurore

1980 Doctrina Christiana : Le Pater Noster. Paris, Société d'ethnographie («Recherches Américaines $2 》$ ).

\section{GIL, Fernando}

1993 Primeras « Doctrinas » del Nuevo Mundo. Estudio histórico-teológico de las obras de fray Juan de Zumarraga (†1548). Buenos Aires, Publicaciones de la facultad de Teología de la Universidad Católica Argentina.

GLASS, John

1975 « A census of Middle American Testerian Manuscripts », in Howard Cline (dir.), Handbook of Middle American Indians. Austin, University of Texas Press : 281-296.

\section{Houston, Stephen}

1994 « Literacy among the Pre-Columbian Maya : A Comparative Perspective », in Elizabeth Hill Boone et Walter D. Mignolo (éd.), Writing Without Words. Alternative Literacies in Mesoamerica and The Andes. Durham-Londres, Duke University Press : 27-49.

\section{KARTTUnen, Frances}

1992 An Analytical Dictionary of Nahuatl. Norman, University of Oklahoma Press.

\section{Kuein, Cecelia F.}

2001 « None of the Above : Gender Ambiguity in Nahua Ideology », in Cecelia F. Klein (éd.), Gender in Pre-Hispanic America. Washington (D.C.), Dumbarton Oaks : 183-253.

\section{LACADENA, Alfonso}

2008 « Regional Scribal Traditions : Methodological Implications for the Decipherment of Nahuatl Writing 》, PARI Journal (numéro spécial « Nahuatl Writing 》) 8(4) : 1-22.

\section{Molina, fray Alonso de}

1889 [1546], « Copia y relación del catecismo de la doctrina cristiana » in J. García Icazbalceta [éd.], Nueva Colección de documentos para la historia de México. T. II. Mexico, Antigua Librería de Andrade y Morales : 33-61.

\section{Normann, Anne Whited}

1985 Testerian Codices: Hieroglyphic Catechisms for Native Conversion in New Spain. La Nouvelle-Orléans, Tulane University, thèse de doctorat.

\section{Orozco y Berra, Manuel}

1877 « Doctrinas en jeroglíficos 》, Anales del Museo Nacional de México 1 : 202-216.

\section{Resines LLORENTE, Luis}

2007 Diccionario de los catecismos pictográficos. Valladolid, Diputación Provincial de Valladolid.

\section{RICARD, Robert}

1933 La Conquête spirituelle du Mexique : essai sur l'apostolat et les méthodes missionnaires des Ordres Mendiants en Nouvelle-Espagne de 1523-24 à 1572. Paris, Institut d'ethnologie.

\section{SAHAGÙn, fray Bernardino de}

1979 Códice florentino. Mexico, Secretaría de Gobernación.

\section{Severi, Carlo}

2007 Le Principe de la chimère. Une anthropologie de la mémoire. Paris, Éditions rue d'Ulm-musée du quai Branly [« Esthetica 》).

\section{Soustelle, Jacques}

1963 « Notas sobre un documento otomí de la Biblioteca Nacional de París », El México antiguo, Revista Internacional de arqueología, etnología, folklore, prehistoria $4(1-2): 13-20$.

\section{VALADÉs, fray Diego de}

1989 [1579] Retórica christiana.

Édité par E. J. Palomera. Mexico, Fondo de cultura económica. 


\section{MANUSCRITS TESTÉRIENS}

806 - «Catecismo de la doctrina cristiana en jeroglificos » ou « catecismo de Pedro de Gante ", Biblioteca Nacional de Madrid, Madrid (fol. 2v, 3r, 4r, 24v, 29r).

807-Mexicain 76, Département des manuscrits, Bibliothèque nationale de France, Paris (fol. 4 r).

808 - Mexicain 77, Département des manuscrits, Bibliothèque nationale de France, Paris (fol. 1r, 1v).

809-Mexicain 78, Département des manuscrits, Bibliothèque nationale de France, Paris (fol. 2v, 3r, 3v, 4r, 7r).

810 - Mexicain 399, Département des manuscrits, Bibliothèque nationale de France, Paris (fol. $3 \mathrm{v}, 4 \mathrm{r}, 4 \mathrm{v}, 5 \mathrm{r}, 7 \mathrm{r}, 11 \mathrm{v}$ ).

813 - Doctrina Cristiana / Egerton Ms. 2898, British Museum, Londres (fol. 1v, 2r, 8r, 10v, 11r).

814 - Phillips Catechism, Fondation Martin Bodmer, Cologny (Suisse) (fol. 1v, 2v, 3r, 9r, 23r).

817-Humboldt Fragment 16 / American Manuscript $n^{\circ} 1$, Deutsche Staatsbibliothek zu Berlin, Berlin.

823 - Wilkinson Ms. 142, Hispanic Society of America, New York (fol. 5v, 6r, 7r).

824 - Codex Indian 25, John Carter Brown Library, Brown University, Providence (fol. 6r).

830 - Codex 35-53 (ou «Libro de oraciones"), Museo Nacional de Antropología, Mexico (fol. 6v, 7r).

832 - Harvard Testerian, Peabody Museum Library, Harvard University, Cambridge (Mass.) (fol. 7r).

833-Garrett Mesoamerican Ms. 3a, Department of Rare Books and Special Collections, Manuscripts Division, Princeton University Library, Princeton (fol. 13r).

\section{Résumé / Abstract}

Bérénice Gaillemin, Images mémorables pour un texte immuable. Les catéchismes pictographiques testériens (Mexique, $x V l^{e}$ $x I x^{e}$ siècles] - Les catéchismes testériens contiennent les prières principales et les différentes listes de préceptes que les missionnaires se devaient d'enseigner afin de convertir et de préparer aux sacrements les nouveaux catéchumènes mexicains. Constitués presque exclusivement d'images, ces petits cahiers étaient censés faciliter la mémorisation de ces textes immuables et pouvaient se substituer à l'apprentissage des caractères latins. Transcription parfois mot à mot des textes catholiques, ces ensembles iconographiques ordonnés offrent une occasion exceptionnelle d'étudier les rapports complexes que les images entretiennent avec la langue et la parole. En postulant que les images peuvent transcrire des mots mais aussi des sons, il est alors possible d'en décrire les principales fonctions sémiotiques. Enfin, il est question de leur rôle comme déclencheur de mémoire, permettant de mieux guider, d'accompagner et de solliciter la participation des lecteurs.

\section{HORS TESTÉRIENS}

Bible historiale, Manuscrit français 20090 (vers 1390), Département des manuscrits, Bibliothèque nationale de France, Paris (fol. 3).

Bible moralisée, 270B (1240), Bodleian Library, Oxford (fol. 34).

Codex Borbonicus, Bibliothèque de l'Assemblée nationale, Paris (pl. 4).

Codex Borgia, Bibliotheca Apostolica Vaticana, Rome (fol. 59).

Codex de Florence, Biblioteca Medicea Laurenziana, Florence (livre 11).

Codex Fejérváry-Mayer, World Museum, Liverpool (pl. 11).

Codex Mendoza, MS. Arch. Selden. A. 1 (vers 1535-1550), Bodleian Library, Oxford (pl. 54, fig. 1, et fol. 13r).

Codex Telleriano-Remensis, Mexicain 385 ,

Département des manuscrits, Bibliothèque nationale de France, Paris.

Moulage de bas-relief, réplique du xIx ${ }^{\mathrm{e}}$ siècle, Peabody Museum, Harvard University, Cambridge (Mass.).

Bérénice Gaillemin, Memorable images for an immutable text. Pictographic Testerian catechisms $\left(16^{\text {th }}\right.$ to $19^{\text {th }}$ Mexico $)$ - Testerian catechisms contain the principal prayers and different lists of precepts that missionaries were meant to teach new Mexican catechumens as they went about converting and preparing them for baptism. These small notebooks were almost entirely composed of images and were intended to help people memorise immutable texts, thereby removing the need for them to learn the Roman alphabet. These ordered iconographic sets are sometimes "word for word" transcriptions of the original Catholic texts, and so represent an exceptional opportunity to study the complex relationships that exist between, on the one hand, images and, on the other, words and speech. If we assume that images can transcribe not only words, but also sounds, then we can begin to describe their principal semiotic functions. The article also explores their role as memory-triggers that allowed missionaries to direct, accompany and encourage the participation of their readers. 


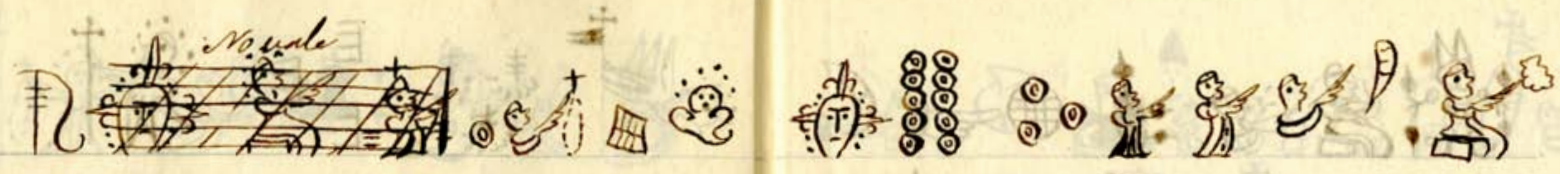

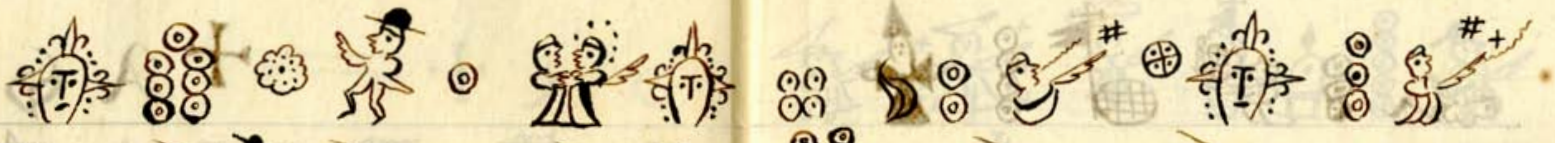
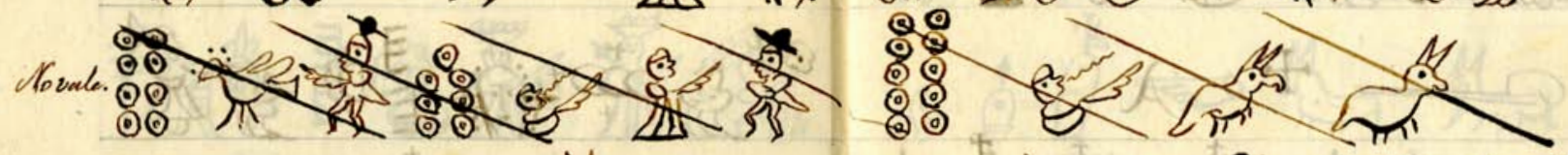

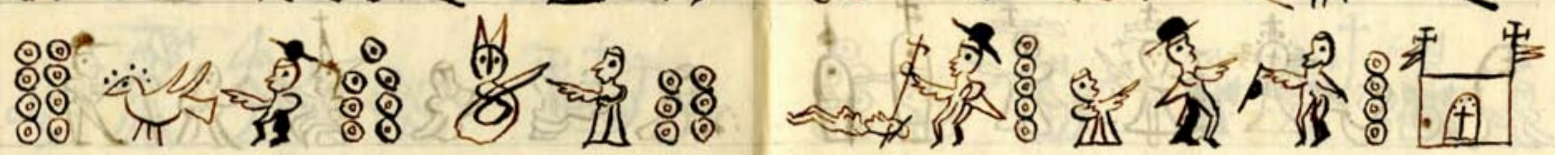

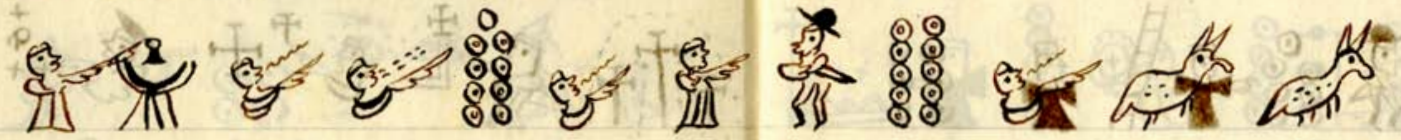

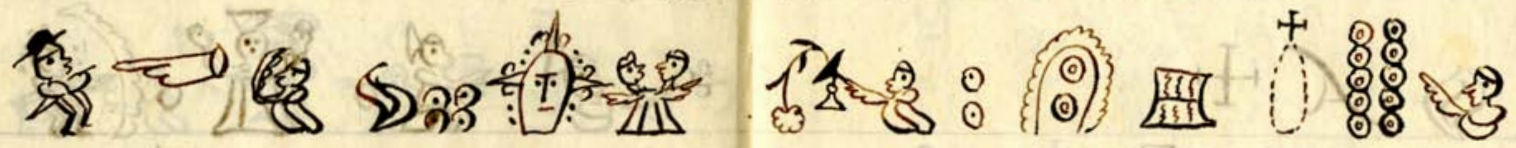

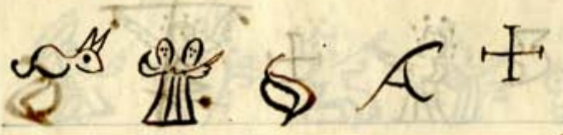

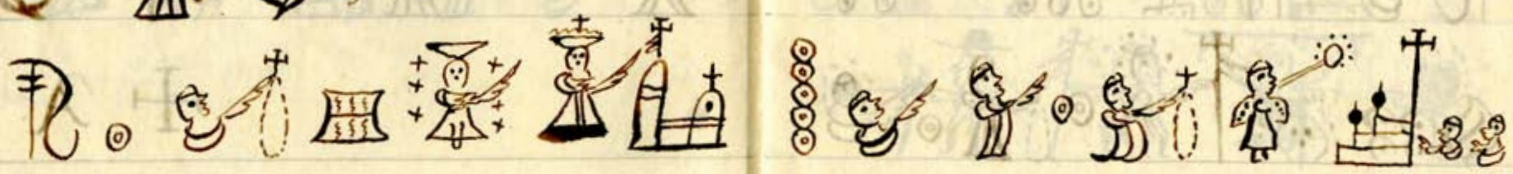

